\title{
Some new Simpson-type inequalities for generalized $p$-convex function on fractal sets with applications
}

\author{
Thabet Abdeljawad ${ }^{1,2,3}$, Saima Rashid ${ }^{4}$, Zakia Hammouch ${ }^{5}$, Imdat İşcan ${ }^{6}$ and Yu-Ming Chu C,8* $^{7}$
}

*Correspondence:

chuyuming2005@126.com

${ }^{7}$ Department of Mathematics,

Huzhou University, Huzhou, China

${ }^{8}$ Hunan Provincial Key Laboratory of

Mathematical Modeling and

Analysis in Engineering, Changsha

University of Science \& Technology,

Changsha, China

Full list of author information is

available at the end of the article

\section{Springer}

\begin{abstract}
The present article addresses the concept of $p$-convex functions on fractal sets. We are able to prove a novel auxiliary result. In the application aspect, the fidelity of the local fractional is used to establish the generalization of Simpson-type inequalities for the class of functions whose local fractional derivatives in absolute values at certain powers are $p$-convex. The method we present is an alternative in showing the classical variants associated with generalized $p$-convex functions. Some parts of our results cover the classical convex functions and classical harmonically convex functions. Some novel applications in random variables, cumulative distribution functions and generalized bivariate means are obtained to ensure the correctness of the present results. The present approach is efficient, reliable, and it can be used as an alternative to establishing new solutions for different types of fractals in computer graphics.
\end{abstract}

MSC: 26D15; 26D10; 90C23

Keywords: Generalized convex function; Generalized s-convex function; Hermite-Hadamard inequality; Simpson's-like type inequality; Generalized $m$-convex functions; Fractal sets

\section{Introduction}

The following inequality is known in the literature as a Simpson-type inequality:

$$
\begin{aligned}
& \left|\frac{1}{6}\left[\chi\left(\varkappa_{1}\right)+4 \chi\left(\frac{\varkappa_{1}+\varkappa_{2}}{2}\right)+\chi\left(\varkappa_{2}\right)\right]-\frac{1}{\varkappa_{2}-\varkappa_{1}} \int_{\varkappa_{1}}^{\varkappa_{2}} \chi(u) d u\right| \\
& \quad \leq \frac{1}{2880}\left\|\chi^{(4)}\right\|_{\infty}\left(\varkappa_{2}-\varkappa_{1}\right)^{4},
\end{aligned}
$$

where the mapping $\chi:\left[\varkappa_{1}, \varkappa_{2}\right] \rightarrow \mathbb{R}$ is assumed to be four times continuously differentiable on the interval $\left(\varkappa_{1}, \varkappa_{2}\right)$ and $\left\|\chi^{(4)}\right\|_{\infty}=\sup _{\xi \in\left(\varkappa_{1}, \varkappa_{2}\right)}\left|\chi^{4}(\xi)\right|<\infty$. The inequality (1.1) has gained considerable attention, as convex analysis and fractional calculus operators involving several classes of convex functions is an uphill task. Therefore many authors proposed different numerical techniques to find Simpson-type inequalities, arising in the substantial literature of numerical analysis and engineering, and many other fields of sci-

(c) The Author(s) 2020. This article is licensed under a Creative Commons Attribution 4.0 International License, which permits use sharing, adaptation, distribution and reproduction in any medium or format, as long as you give appropriate credit to the original author(s) and the source, provide a link to the Creative Commons licence, and indicate if changes were made. The images or other third party material in this article are included in the article's Creative Commons licence, unless indicated otherwise in a credit line to the material. If material is not included in the article's Creative Commons licence and your intended use is not permitted by statutory regulation or exceeds the permitted use, you will need to obtain permission directly from the copyright holder. To view a copy of this licence, visit http://creativecommons.org/licenses/by/4.0/. 
ences [1-12]. Profusely novel versions of Simpson-type inequalities for the class of convex functions have been modified and generalized by numerous researchers [13-24]. Recently, many investigations about (1.1) can be found by Rashid et al. [25] for preinvex functions, $\mathrm{Li}$ and $\mathrm{Du}[26]$ for $(\dot{\alpha}, m)$-GA-convex functions, Xi and Qi [27] for logarithmically convex functions, Sarikaya et al. [28] for $s$-convex functions and İşcan et al. [29] for $p$-convex function.

With the development of the inequality theory, the inequalities for various kinds of convex functions have a rapid blossom in the area of pure and applied mathematics [30-42]. As mentioned above, many articles are all involved with Hermite-Hadamard type and trapezoid type, midpoint type inequalities [43-49]. However, to the best of our knowledge, Simpson-type inequalities for functions whose first local $\dot{\alpha}$-derivatives in absolute value are the class of generalized $p$-convex functions have not been reported. So we turn our attention to this new research.

One of the aspects which are nowadays particularly well known among researchers is the integral inequalities with applications. In this field, the majority of the authors are generalizing the standard results in the accessible literature by utilizing various sorts/definitions of the fractional integral operators $[4,25,50,51]$. An enormous heft of fractional differential problems and partial differential equations can be converted into problems of comprehending some estimated integral equations.

The early research motivations in the area of the local fractional theory were for solving a bulk of initial and boundary value associated with differential equations [52, 53]. In [53], Yang introduced a contemporary study used to tackle nondifferentiable problems that incorporate in complex systems of real-world phenomena. The fractal sets in science have introduced some fascinating complex graphs and picture compressions to computer graphics. The expression "fractal" was first utilized by a young mathematician, Julia [54] when he was considering Cayley's problem identified with the conduct of Newton's method in a complex plane. The fractal is frequently utilized in real-world studies, involving fractal antennas, fractal transistors, and fractal heat exchangers. It has applications in the music industry, the creation of photography, soil mechanics, small-angle scattering theory, and many more fileds. It is to be emphasized that fractal theory assumes an essential job in the improvement of picturing of fractal sets. The utilizations of fractal sets in cryptography and other useful areas of research have increased the interest of researchers to broaden the utilization in mathematical inequalities. Fractals are particular arbitrary examples descriptions addressing the erratic developments of the disorderly world at work. The most significant utilization of fractals in software engineering is fractal picture compression. This sort of compression utilizes the way that this present reality is very well portrayed by fractal geometry [52, 55, 56]. Interestingly, the author of [53] investigated the local fractional functions on fractal space deliberately, which comprises of local fractional calculus and the monotonicity of functions. Numerous analysts contemplated the characteristics of functions on fractal space and built numerous sorts of fractional calculus by utilizing various strategies [57-59]. Additionally, integral inequalities in the context of local fractional calculus have a significant role in all fields of pure and applied mathematics. For example, Chen [60] derived a novel version of Hölder inequality on fractals. In [58], Mo et al. established the fractal version of Hermite-Hadamard inequality by the use of generalized convex functions. In [61], Du et al. contemplated the novel generalizations for Simpson's, Hermite Hadamard and Hermite-Hadamard-Fejér type inequalities 
for generalized $m$-convex functions concerning to local fractional calculus. In addition to these results, Luo et al. [62], deduced several new Fejér-Hermite-Hadamard inequalities for a class of $h$-convex functions with applications. For some useful and recent studies on fractional calculus and its applications in different fields of mathematics [63-68].

Owing to the above phenomena, the key aim of this research is to introduce a new auxiliary result depending on local fractal sets are presented. With the aid of novel identity, we derived numerous novel generalizations of Simpson-type for mappings whose powers contain local fractional derivatives in modulus are generalized $p$-convex. The main impetus of this study to capture new estimates for generalized convex functions and generalized harmonically convex functions. In addition, the application of the proved results in a random variable, cumulative distribution functions, and the generalized bivariate mean formula is also presented. We hope that the new strategy formulated in the present paper is more invigorating than the accessible one.

\section{Preliminaries}

Now, we mention the preliminaries from the theory of local fractional calculus. These ideas and important consequences associated with the local fractional derivative and local fractional integral are mainly due to Yang et al. [53].

Let $\varsigma_{1}^{\dot{\alpha}}, \varsigma_{2}^{\dot{\alpha}}$ and $\varsigma_{3}^{\dot{\alpha}}$ belong to the set $\mathbb{R}^{\dot{\alpha}}(0<\dot{\alpha} \leq 1)$. Then

(1) $\varsigma_{1}^{\dot{\alpha}}+\varsigma_{2}^{\dot{\alpha}}$ and $\varsigma_{1}^{\dot{\alpha}} \zeta_{2}^{\dot{\alpha}}$ belong to the set $\mathbb{R}^{\dot{\alpha}}$;

(2) $\varsigma_{1}^{\dot{\alpha}}+\varsigma_{2}^{\dot{\alpha}}=\varsigma_{2}^{\dot{\alpha}}+\varsigma_{1}^{\dot{\alpha}}=\left(\varsigma_{1}+\varsigma_{2}\right)^{\dot{\alpha}}=\left(\varsigma_{2}+\varsigma_{1}\right)^{\dot{\alpha}}$;

(3) $\varsigma_{1}^{\dot{\alpha}}+\left(\varsigma_{2}^{\dot{\alpha}}+\varsigma_{3}^{\dot{\alpha}}\right)=\left(\varsigma_{1}^{\dot{\alpha}}+\varsigma_{2}^{\dot{\alpha}}\right)+\varsigma_{3}^{\dot{\alpha}}$;

(4) $\varsigma_{1}^{\dot{\alpha}} \varsigma_{2}^{\dot{\alpha}}=\varsigma_{2}^{\dot{\alpha}} \varsigma_{1}^{\dot{\alpha}}=\left(\varsigma_{1} \varsigma_{2}\right)^{\dot{\alpha}}=\left(\varsigma_{2} \varsigma_{1}\right)^{\dot{\alpha}}$;

(5) $\varsigma_{1}^{\dot{\alpha}}\left(\varsigma_{2}^{\dot{\alpha}} \varsigma_{3}^{\dot{\alpha}}\right)=\left(\varsigma_{1}^{\dot{\alpha}} \varsigma_{2}^{\dot{\alpha}}\right) \varsigma_{3}^{\dot{\alpha}}$;

(6) $\varsigma_{1}^{\dot{\alpha}}\left(\varsigma_{2}^{\dot{\alpha}}+\varsigma_{3}^{\dot{\alpha}}\right)=\varsigma_{1}^{\dot{\alpha}} \varsigma_{2}^{\dot{\alpha}}+\varsigma_{1}^{\dot{\alpha}} \varsigma_{3}^{\dot{\alpha}}$;

(7) $\varsigma_{1}^{\dot{\alpha}}+0^{\dot{\alpha}}=0^{\dot{\alpha}}+\zeta_{1}^{\dot{\alpha}}=\zeta_{1}^{\dot{\alpha}}$ and $\zeta_{1}^{\dot{\alpha}} 1^{\dot{\alpha}}=1^{\dot{\alpha}} \zeta_{1}^{\dot{\alpha}}=\zeta_{1}^{\dot{\alpha}}$.

Definition 2.1 A nondifferentiable mapping $\chi: \mathbb{R} \rightarrow \mathbb{R}^{\dot{\alpha}}, \theta \rightarrow \chi(\epsilon)$ is said to be local fractional continuous at $\epsilon_{\circ}$, if, for any $\epsilon>0$, there exists $\xi>0$ such that

$$
\left|\chi(\epsilon)-\chi\left(\epsilon_{\circ}\right)\right|<\epsilon^{\dot{\alpha}}
$$

for $\left|\epsilon-\epsilon_{0}\right|<\kappa$. If $\chi(\epsilon)$ is local continuous on $\left(\varkappa_{1}, \varkappa_{2}\right)$, then we denote it by $\chi(\epsilon) \epsilon$ $\mathbb{C}_{\dot{\alpha}}\left(\varkappa_{1}, \varkappa_{2}\right)$.

Definition 2.2 The local fractional derivative of $\chi(\epsilon)$ of order $\dot{\alpha}$ at $\epsilon=\epsilon_{\circ}$ is defined by the expression

$$
\begin{aligned}
\chi^{(\dot{\alpha})}\left(\epsilon_{\circ}\right) & =\epsilon_{\circ} \mathcal{D}_{\epsilon}^{\dot{\alpha}} \chi(\epsilon)=\left.\frac{d^{\dot{\alpha}} \chi(\epsilon)}{d \epsilon^{\dot{\alpha}}}\right|_{\epsilon=\epsilon_{\circ}} \\
& =\lim _{\epsilon \rightarrow \epsilon_{\circ}} \frac{\Delta^{\dot{\alpha}}\left(\chi(\epsilon)-\chi\left(\epsilon_{\circ}\right)\right)}{\left(\epsilon-\epsilon_{\circ}\right)^{\dot{\alpha}}},
\end{aligned}
$$

where $\Delta^{\dot{\alpha}}\left(\chi(\epsilon)-\chi\left(\epsilon_{\circ}\right)\right)=\Gamma(\dot{\alpha}+1)\left(\chi(\epsilon)-\chi\left(\epsilon_{\circ}\right)\right)$. Let $\chi^{(\dot{\alpha})}(\epsilon)=\mathcal{D}_{\epsilon}^{\dot{\alpha}} \chi(\epsilon)$. If there exists

$\chi^{(k+1) \dot{\alpha}}(\epsilon)=\overbrace{\mathcal{D}_{\epsilon}^{\dot{\alpha}} \ldots \mathcal{D}_{\epsilon}^{\dot{\alpha}}}^{(k+1) \text { times }}(\epsilon)$ for any $\epsilon \in \Omega \subseteq \mathbb{R}$, then it is denoted by $\chi \in \mathcal{D}_{(k+1) \dot{\alpha}}(\mathcal{I})$, where $k=0,1,2, \ldots$. 
Definition 2.3 Let $\chi(\epsilon) \in \mathbb{C}_{\dot{\alpha}}\left[\varkappa_{1}, \varkappa_{2}\right]$ and $\Delta=\left\{\xi_{0}, \xi_{1}, \ldots, \xi_{N}\right\}(N \in \mathbb{N})$ be a partition of $\left[\varkappa_{1}, \varkappa_{2}\right]$ with $\varkappa_{1}=\xi_{0}<\xi_{1}<\cdots<\xi_{N}=\varkappa_{2}$. Then the local fractional integral of $\chi$ on $\left[\varkappa_{1}, \varkappa_{2}\right]$ of order $\dot{\alpha}$ is defined by

$$
\begin{aligned}
\varkappa_{1} \mathcal{I}_{\varkappa_{2}}^{(\dot{\alpha})} \chi(\epsilon) & =\frac{1}{\Gamma(1+\dot{\alpha})} \int_{\varkappa_{1}}^{\varkappa_{2}} \chi(\xi)(d \xi)^{\dot{\alpha}} \\
& :=\frac{1}{\Gamma(1+\dot{\alpha})} \lim _{\delta \xi \rightarrow 0} \sum_{j=0}^{N-1} \chi\left(\xi_{j}\right)\left(\Delta \xi_{j}\right),
\end{aligned}
$$

where $\delta \xi:=\max \left\{\Delta \xi_{1}, \Delta \xi_{2}, \ldots, \Delta \xi_{N-1}\right\}$ and $\Delta \xi_{j}:=\xi_{j+1}-\xi_{j}, j=0, \ldots, N-1$.

We clearly see that ${ }_{\varkappa_{1}} \mathcal{I}_{\varkappa_{2}}^{(\dot{\alpha})} \chi(\epsilon)=0$ if $\varkappa_{1}=\varkappa_{2}$ and ${ }_{\varkappa_{1}} \mathcal{I}_{\varkappa_{2}}^{(\dot{\alpha})} \chi(\epsilon)=-\varkappa_{2} \mathcal{I}_{\varkappa_{1}}^{(\dot{\alpha})} \chi(\epsilon)$ if $\varkappa_{1}<\varkappa_{2}$. For any $\epsilon \in\left[\varkappa_{1}, \varkappa_{2}\right]$, if there exists $\varkappa_{1} \mathcal{I}_{\varkappa_{2}(\dot{\alpha})} \chi(\epsilon)$, then it is denoted by $\chi(\epsilon) \in \mathcal{I}_{\epsilon}^{\dot{\alpha}}\left[\varkappa_{1}, \varkappa_{2}\right]$.

Lemma 2.4 (See [53]) The following statements are true:

(1) If $\chi(u)=\mathcal{G}^{(\dot{\alpha})}(u) \in \mathbb{C}_{\dot{\alpha}}\left[\varkappa_{1}, \varkappa_{2}\right]$, then

$$
\varkappa_{1} \mathcal{I}_{\varkappa_{2}}^{(\dot{\alpha})} \chi(u)=\mathcal{G}\left(\varkappa_{2}\right)-\mathcal{G}\left(\varkappa_{1}\right)
$$

(2) If $\chi(u), \mathcal{G}(u) \in \mathcal{D}_{\dot{\alpha}}\left[\varkappa_{1}, \varkappa_{2}\right]$, and $\chi^{(\dot{\alpha})}(u), \mathcal{G}^{(\dot{\alpha})}(u) \in \mathbb{C}_{\dot{\alpha}}\left[\varkappa_{1}, \varkappa_{2}\right]$, then

$$
{ }_{\varkappa_{1}} \mathcal{I}_{\varkappa_{2}}^{(\dot{\alpha})} \chi(u) \mathcal{G}^{(\dot{\alpha})}(u)=\left.\chi(u) \mathcal{G}(u)\right|_{\varkappa_{1}} ^{\varkappa_{2}}-\varkappa_{1} \mathcal{I}_{\varkappa_{2}}^{(\dot{\alpha})} \chi^{(\dot{\alpha})}(u) \mathcal{G}(u)
$$

Lemma 2.5 (See [53]) The formulas

$$
\begin{aligned}
& \frac{d^{\dot{\alpha}} u^{k \dot{\alpha}}}{d u^{\dot{\alpha}}}=\frac{\Gamma(1+k \dot{\alpha})}{\Gamma(1+(k-1) \dot{\alpha})} u^{(k-1) \dot{\alpha}}, \\
& \frac{1}{\Gamma(1+\dot{\alpha})} \int_{\varkappa_{1}}^{\varkappa_{2}} u^{k \dot{\alpha}}(d u)^{\dot{\alpha}}=\frac{\Gamma(1+k \dot{\alpha})}{\Gamma(1+(k+1) \dot{\alpha})}\left(\varkappa_{2}^{(k+1) \dot{\alpha}}-\varkappa_{1}^{(k+1) \dot{\alpha}}\right),
\end{aligned}
$$

hold for $k>0$.

Lemma 2.6 (Generalized Hölder inequality [60]) Let $s, q>1$ with $s^{-1}+q^{-1}=1$ and $\chi, \mathcal{G} \in$ $\mathbb{C}_{\dot{\alpha}}\left[\varkappa_{1}, \varkappa_{2}\right]$. Then

$$
\begin{aligned}
& \frac{1}{\Gamma(1+\dot{\alpha})} \int_{\varkappa_{1}}^{\varkappa_{2}}|\chi(u) \mathcal{G}(u)|(d u)^{\dot{\alpha}} \\
& \quad \leq\left(\frac{1}{\Gamma(1+\dot{\alpha})} \int_{\varkappa_{1}}^{\varkappa_{2}}|\chi(u)|^{s}(d u)^{\dot{\alpha}}\right)^{\frac{1}{s}}\left(\frac{1}{\Gamma(1+\dot{\alpha})} \int_{\varkappa_{1}}^{\varkappa_{2}}|\mathcal{G}(u)|^{q}(d u)^{\dot{\alpha}}\right)^{\frac{1}{q}} .
\end{aligned}
$$

\section{Main results and discussions}

In this section, we first inaugurate a local fractional integral identity for generalized $p$ convex functions, and then we utilize the said identity to establish certain Simpson-type variants in the context of the fractal domain. We now present the concept of generalized $p$-convex functions on fractal space as follows. 
Definition 3.1 Let $p \in \mathbb{R} \backslash\{0\}$. Then $\chi: \Omega=\left[\varkappa_{1}, \varkappa_{2}\right] \rightarrow \mathbb{R}^{\dot{\alpha}}$ is said to be generalized $p$ convex if the inequality

$$
\chi\left(\left[(1-\xi) x^{p}+\xi y^{p}\right]^{\frac{1}{p}}\right) \leq(1-\xi)^{\dot{\alpha}} \chi(x)+\xi^{\dot{\alpha}} \chi(y)
$$

holds for $x, y \in \Omega$, and $\xi \in[0,1] . \chi$ is said to be generalized $p$-concave if inequality (3.1) is reversed.

Remark 3.2 From Definition 3.1 we clearly see that:

(1) If we take $\dot{\alpha}=1$, then we get a definition given in [69].

(2) If we take $p=1$, then we get a definition given in [58].

(3) If we take $p=-1$, then we get a definition given in [66].

(4) If we take $p=1$ with $\dot{\alpha}=1$, then we get the classical convex function.

(5) If we take $p=-1$ and $\dot{\alpha}=1$, then we get a definition given in [70].

It is worth mentioning that generalized $p$-convex functions collapse to generalized convex, generalized harmonically convex functions, harmonically convex functions, and classical convex functions as special cases. This shows that outcomes derived in the present paper continue to hold for these classes of convex functions and their variant forms.

Example 3.3 Let $\chi: \Omega=\left[\varkappa_{1}, \varkappa_{2}\right] \rightarrow \mathbb{R}^{\dot{\alpha}}$ with $\chi_{1}(u)=u^{\dot{\alpha} p}(p \neq 0)$ and $\chi_{2}: \Omega \rightarrow \mathbb{R}^{\dot{\alpha}}$ with $\chi_{2}(u)=c^{\dot{\alpha}}\left(c^{\dot{\alpha}} \in \mathbb{R}^{\dot{\alpha}}\right)$. Then $\chi_{1}$ is generalized $p$-convex and $\chi_{2}$ is generalized $p$-concave.

Lemma 3.4 Let $p \in \mathbb{R} \backslash\{0\}$ and $\chi: \Omega^{\circ} \subset \mathbb{R} \rightarrow \mathbb{R}^{\dot{\alpha}}$ such that $\chi \in \mathcal{D}_{\dot{\alpha}}\left(\Omega^{\circ}\right)\left(\Omega^{\circ}\right.$ is the interior of $\Omega$ ) and $\chi^{(\dot{\alpha})} \in \mathcal{C}_{\dot{\alpha}}\left[\varkappa_{1}, \varkappa_{2}\right]$. Then the inequality

$$
\begin{aligned}
\left(\frac{1}{6}\right)^{\dot{\alpha}} & {\left[\chi\left(\varkappa_{1}\right)+(4)^{\dot{\alpha}} \chi\left(\left[\frac{\varkappa_{1}^{p}+\varkappa_{2}^{p}}{2}\right]^{1 / p}\right)+\chi\left(\varkappa_{2}\right)\right] } \\
& -\left(\frac{2 p}{\varkappa_{2}^{p}-\varkappa_{1}^{p}}\right)^{\dot{\alpha}} \varkappa_{1} \mathcal{I}_{\varkappa_{2}}^{(\dot{\alpha})} \frac{\chi(u)}{u^{\dot{\alpha}(1-p)}} \\
\leq & \left(\frac{\varkappa_{2}^{p}-\varkappa_{1}^{p}}{4 p}\right)^{\dot{\alpha}}\left[\frac{1}{\Gamma(1+\dot{\alpha})} \int_{0}^{1} \frac{\left(\xi-\frac{1}{3}\right)^{\dot{\alpha}}}{\left[(1-\xi) \varkappa_{1}^{p}+\xi \frac{\varkappa_{1}^{p}+\varkappa_{2}^{p}}{2}\right]^{\dot{\alpha}(1-1 / p)}}\right] \\
& \times \chi^{(\dot{\alpha})}\left(\left[(1-\xi) \varkappa_{1}^{p}+\xi \frac{\varkappa_{1}^{p}+\varkappa_{2}^{p}}{2}\right]^{1 / p}\right)(d \xi)^{\dot{\alpha}} \\
& +\left(\frac{\varkappa_{2}^{p}-\varkappa_{1}^{p}}{4 p}\right)^{\dot{\alpha}}\left[\frac{1}{\Gamma(1+\dot{\alpha})} \int_{0}^{1} \frac{\left(\xi-\frac{2}{3}\right)^{\dot{\alpha}}}{\left[(1-\xi) \frac{\varkappa_{1}^{p}+\varkappa_{2}^{p}}{2}+\xi \varkappa_{2}^{p}\right]^{\dot{\alpha}(1-1 / p)}}\right] \\
& \times \chi^{(\dot{\alpha})}\left(\left[(1-\xi) \frac{\varkappa_{1}^{p}+\varkappa_{2}^{p}}{2}+\xi \varkappa_{2}^{p}\right]^{1 / p}\right)(d \xi)^{\dot{\alpha}}
\end{aligned}
$$

holds for all $u \in\left[\varkappa_{1}, \varkappa_{2}\right]$.

Proof Firstly, let us calculate the following integrals:

$$
\left(\frac{\varkappa_{2}^{p}-\varkappa_{1}^{p}}{4 p}\right)^{\dot{\alpha}}\left[\frac{1}{\Gamma(1+\dot{\alpha})} \int_{0}^{1} \frac{\left(\xi-\frac{1}{3}\right)^{\dot{\alpha}}}{\left[(1-\xi) \varkappa_{1}^{p}+\xi \frac{\varkappa_{1}^{p}+\varkappa_{2}^{p}}{2}\right]^{\dot{\alpha}(1-1 / p)}}\right]
$$




$$
\begin{aligned}
& \times \chi^{(\dot{\alpha})}\left(\left[(1-\xi) \varkappa_{1}^{p}+\xi \frac{\varkappa_{1}^{p}+\varkappa_{2}^{p}}{2}\right]^{1 / p}\right)(d \xi)^{\dot{\alpha}} \\
& +\left(\frac{\varkappa_{2}^{p}-\varkappa_{1}^{p}}{4 p}\right)^{\dot{\alpha}}\left[\frac{1}{\Gamma(1+\dot{\alpha})} \int_{0}^{1} \frac{\left(\xi-\frac{2}{3}\right)^{\dot{\alpha}}}{\left[(1-\xi) \frac{\varkappa_{1}^{p}+\varkappa_{2}^{p}}{2}+\xi \varkappa_{2}^{p}\right]^{\dot{\alpha}(1-1 / p)}}\right] \\
& \times \chi^{(\dot{\alpha})}\left(\left[(1-\xi) \frac{\varkappa_{1}^{p}+\varkappa_{2}^{p}}{2}+\xi \varkappa_{2}^{p}\right]^{1 / p}\right)(d \xi)^{\dot{\alpha}}:=I_{1}+I_{2} .
\end{aligned}
$$

Utilizing the local fractional integration by parts and the change of the variable technique for the integrals $I_{1}$ and $I_{2}$, we get

$$
\begin{aligned}
& I_{1}=\left[\int_{0}^{1} \frac{\left(\xi-\frac{1}{3}\right)^{\dot{\alpha}}}{\left[(1-\xi) \varkappa_{1}^{p}+\xi \frac{\varkappa_{1}^{p}+\varkappa_{2}^{p}}{2}\right]^{\dot{\alpha}(1-1 / p)}}\right] \chi^{(\dot{\alpha})}\left(\left[(1-\xi) \varkappa_{1}^{p}+\xi \frac{\varkappa_{1}^{p}+\varkappa_{2}^{p}}{2}\right]^{1 / p}\right)(d \xi)^{\dot{\alpha}} \\
& =\left.\left(\xi-\frac{1}{3}\right)^{\dot{\alpha}} \chi\left(\left[(1-\xi) \varkappa_{1}^{p}+\xi \frac{\varkappa_{1}^{p}+\varkappa_{2}^{p}}{2}\right]^{1 / p}\right)\right|_{0} ^{1} \\
& -\left(\frac{2 p}{\varkappa_{2}^{p}-\varkappa_{1}^{p}}\right)^{\dot{\alpha}} \frac{\Gamma(1+\dot{\alpha})}{\Gamma(1+\dot{\alpha})} \int_{0}^{1} \chi\left(\left[(1-\xi) \varkappa_{1}^{p}+\xi \frac{\varkappa_{1}^{p}+\varkappa_{2}^{p}}{2}\right]^{1 / p}\right)(d \xi) \\
& =\left(\frac{2}{3}\right)^{\dot{\alpha}} \chi\left(\left[\frac{\varkappa_{1}^{p}+\varkappa_{2}^{p}}{2}\right]^{1 / p}\right)-\left(-\frac{1}{3}\right)^{\dot{\alpha}} \chi\left(\varkappa_{1}\right) \\
& -\left(\frac{2 p}{\varkappa_{2}^{p}-\varkappa_{1}^{p}}\right)^{\dot{\alpha}} \frac{\Gamma(1+\dot{\alpha})}{\Gamma(1+\dot{\alpha})} \int_{\varkappa_{1}}^{\left(\frac{\varkappa_{1}^{p}+\varkappa_{2}^{p}}{2}\right)^{1 / p}} \frac{\chi(u)}{u^{\dot{\alpha}(1-p)}}(d u)^{\dot{\alpha}} \\
& =\left(\frac{2}{3}\right)^{\dot{\alpha}} \chi\left(\left[\frac{\varkappa_{1}^{p}+\varkappa_{2}^{p}}{2}\right]^{1 / p}\right)-\left(-\frac{1}{3}\right)^{\dot{\alpha}} \chi\left(\varkappa_{1}\right) \\
& -\left(\frac{2 p}{\varkappa_{2}^{p}-\varkappa_{1}^{p}}\right)^{\dot{\alpha}} \Gamma(1+\dot{\alpha})_{\varkappa_{1}} \mathcal{I}_{\left(\frac{\varkappa_{1}^{p}+\varkappa_{2}^{p}}{2}\right)^{1 / p}}^{\left(\dot{p} u^{\dot{\alpha}(1-p)}\right.}
\end{aligned}
$$

and

$$
\begin{aligned}
I_{2}= & {\left[\frac{1}{\Gamma(1+\dot{\alpha})} \int_{0}^{1} \frac{\left(\xi-\frac{2}{3}\right)^{\dot{\alpha}}}{\left[(1-\xi) \frac{\varkappa_{1}^{p}+\varkappa_{2}^{p}}{2}+\xi \varkappa_{2}^{p}\right]^{\dot{\alpha}(1-1 / p)}}\right] } \\
& \times \chi^{(\dot{\alpha})}\left(\left[(1-\xi) \frac{\varkappa_{1}^{p}+\varkappa_{2}^{p}}{2}+\xi \varkappa_{2}^{p}\right]^{1 / p}\right)(d \xi)^{\dot{\alpha}} \\
= & \left.\left(\xi-\frac{2}{3}\right)^{\dot{\alpha}} \chi^{(\dot{\alpha})}\left(\left[(1-\xi) \frac{\varkappa_{1}^{p}+\varkappa_{2}^{p}}{2}+\xi \varkappa_{2}^{p}\right]^{1 / p}\right)\right|_{0} ^{1} \\
& -\left(\frac{2 p}{\varkappa_{2}^{p}-\varkappa_{1}^{p}}\right)^{\dot{\alpha}} \int_{0}^{1} \chi^{(\dot{\alpha})}\left(\left[(1-\xi) \frac{\varkappa_{1}^{p}+\varkappa_{2}^{p}}{2}+\xi \varkappa_{2}^{p}\right]^{1 / p}\right)(d \xi)^{\dot{\alpha}} \\
= & \left(\frac{1}{3}\right)^{\dot{\alpha}} \chi\left(\varkappa_{2}\right)-\left(-\frac{2}{3}\right)^{\dot{\alpha}} \chi\left(\left[\frac{\varkappa_{1}^{p}+\varkappa_{2}^{p}}{2}\right]^{1 / p}\right) \\
& -\left(\frac{2 p}{\varkappa_{2}^{p}-\varkappa_{1}^{p}}\right)^{\dot{\alpha}} \frac{\Gamma(1+\dot{\alpha})}{\Gamma(1+\dot{\alpha})} \int_{\left(\frac{\varkappa_{1}^{p}+\varkappa_{2}^{p}}{2}\right)^{1 / p} \frac{\chi(u)}{\varkappa^{\dot{\alpha}(1-p)}}(d u)^{\dot{\alpha}}}
\end{aligned}
$$




$$
\begin{aligned}
= & \left(\frac{1}{3}\right)^{\dot{\alpha}} \chi\left(\varkappa_{2}\right)-\left(-\frac{2}{3}\right)^{\dot{\alpha}} \chi\left(\left[\frac{\varkappa_{1}^{p}+\varkappa_{2}^{p}}{2}\right]^{1 / p}\right) \\
& -\left(\frac{2 p}{\varkappa_{2}^{p}-\varkappa_{1}^{p}}\right)^{\dot{\alpha}} \Gamma(1+\dot{\alpha})_{\left(\frac{\varkappa_{1}^{p}+\varkappa_{2}^{p}}{2}\right)^{1 / p}} \mathcal{I}_{\varkappa_{2}}^{(\dot{\alpha})} \frac{\chi(u)}{u^{\dot{\alpha}(1-p)}} .
\end{aligned}
$$

Adding (3.2) and (3.3) and then multiplying the obtained result by $\frac{1}{2^{\alpha}}$, we have

$$
\begin{aligned}
\frac{I_{1}+I_{2}}{2^{\dot{\alpha}}}= & \left(\frac{1}{6}\right)^{\dot{\alpha}}\left[\chi\left(\varkappa_{1}\right)+(4)^{\dot{\alpha}} \chi\left(\left[\frac{\varkappa_{1}^{p}+\varkappa_{2}^{p}}{2}\right]^{1 / p}\right)+\chi\left(\varkappa_{2}\right)\right] \\
& -\left(\frac{2 p}{\varkappa_{2}^{p}-\varkappa_{1}^{p}}\right)^{\dot{\alpha}} \varkappa_{1} \mathcal{I}_{\varkappa_{2}}^{(\dot{\alpha})} \frac{\chi(u)}{u^{\dot{\alpha}(1-p)}} .
\end{aligned}
$$

Equation (3.4) gives the desired result.

Theorem 3.5 Let $p \in \mathbb{R} \backslash\{0\}, s, q>1$ with $s^{-1}+q^{-1}=1$ and $\chi: \Omega^{\circ} \subset \mathbb{R} \rightarrow \mathbb{R}^{\dot{\alpha}}$ such that $\chi \in \mathcal{D}_{\dot{\alpha}}\left(\Omega^{\circ}\right)\left(\Omega^{\circ}\right.$ is the interior of $\left.\Omega\right), \chi^{(\dot{\alpha})} \in \mathcal{C}_{\dot{\alpha}}\left[\varkappa_{1}, \varkappa_{2}\right]$ and $\left|\chi^{(\dot{\alpha})}\right|^{q}$ is a generalized $p$-convex function on $\Omega$. Then one has

$$
\begin{aligned}
\mid\left(\frac{1}{6}\right)^{\dot{\alpha}} & {\left[\chi\left(\varkappa_{1}\right)+(4)^{\dot{\alpha}} \chi\left(\left[\frac{\varkappa_{1}^{p}+\varkappa_{2}^{p}}{2}\right]^{1 / p}\right)+\chi\left(\varkappa_{2}\right)\right] } \\
& -\left(\frac{2 p}{\varkappa_{2}^{p}-\varkappa_{1}^{p}}\right)^{\dot{\alpha}} \varkappa_{1} \mathcal{I}_{\varkappa_{2}}^{(\dot{\alpha})} \frac{\chi(u)}{u^{\dot{\alpha}(1-p)}} \mid \\
\leq & \left(\frac{\varkappa_{2}^{p}-\varkappa_{1}^{p}}{4 p}\right)^{\dot{\alpha}}\left[\left(\Lambda_{1}^{(\dot{\alpha})}\left(\varkappa_{1},\left(\frac{\varkappa_{1}^{p}+\varkappa_{2}^{p}}{2}\right)^{1 / p} ; p\right)\right)^{1-\frac{1}{q}}\right. \\
& \times\left[\Lambda_{2}^{(\dot{\alpha})}\left(\varkappa_{1},\left(\frac{\varkappa_{1}^{p}+\varkappa_{2}^{p}}{2}\right)^{1 / p} ; p\right)\left|\chi^{(\dot{\alpha})}\left(\varkappa_{1}\right)\right|^{q}\right. \\
& \left.+\Lambda_{3}^{(\dot{\alpha})}\left(\varkappa_{1},\left(\frac{\varkappa_{1}^{p}+\varkappa_{2}^{p}}{2}\right)^{1 / p} ; p\right)\left|\chi^{(\dot{\alpha})}\left(\frac{\varkappa_{1}^{p}+\varkappa_{2}^{p}}{2}\right)^{1 / p}\right|^{q}\right]^{\frac{1}{q}} \\
& +\left(\Lambda_{4}^{(\dot{\alpha})}\left(\left(\frac{\varkappa_{1}^{p}+\varkappa_{2}^{p}}{2}\right)^{1 / p}, \varkappa_{2} ; p\right)\right)^{1-\frac{1}{q}} \\
& \times\left[\Lambda_{5}^{(\dot{\alpha})}\left(\left(\frac{\varkappa_{1}^{p}+\varkappa_{2}^{p}}{2}\right)^{1 / p}, \varkappa_{2} ; p\right)\left|\chi^{(\dot{\alpha})}\left(\frac{\varkappa_{1}^{p}+\varkappa_{2}^{p}}{2}\right)^{1 / p}\right|^{q}\right. \\
& \left.\left.+\Lambda_{6}^{(\dot{\alpha})}\left(\left(\frac{\varkappa_{1}^{p}+\varkappa_{2}^{p}}{2}\right)^{1 / p}, \varkappa_{2} ; p\right)\left|\chi^{(\dot{\alpha})}\left(\varkappa_{2}\right)\right|^{q}\right]^{\frac{1}{q}}\right],
\end{aligned}
$$

where

$$
\begin{aligned}
\Lambda_{1}^{(\dot{\alpha})} & \left(\varkappa_{1},\left(\frac{\varkappa_{1}^{p}+\varkappa_{2}^{p}}{2}\right)^{1 / p} ; p\right) \\
:= & \left(\frac{2}{3}\right)^{\dot{\alpha}}\left(\frac{p}{\left(\varkappa_{2}^{p}-\varkappa_{1}^{p}\right)^{2}}\right)^{\dot{\alpha}}\left[\frac{\left(\varkappa_{1}^{p}+\varkappa_{2}^{p}\right)^{\dot{\alpha}}}{\Gamma(1+\dot{\alpha})}\left\{\left(\frac{5 \varkappa_{1}^{p}+\varkappa_{2}^{p}}{6}\right)^{\frac{1}{p}}-\varkappa_{1}\right)^{\dot{\alpha}}\right. \\
& \left.-\left(\left(\frac{\varkappa_{1}^{p}+\varkappa_{2}^{p}}{2}\right)^{1 / p}-\left(\frac{5 \varkappa_{1}^{p}+\varkappa_{2}^{p}}{6}\right)^{\frac{1}{p}}\right)^{\dot{\alpha}}\right\}-\frac{6^{\dot{\alpha}} \Gamma(1+p \dot{\alpha})}{\Gamma(1+(p+1) \dot{\alpha})}
\end{aligned}
$$




$$
\begin{aligned}
& \left.\times\left\{\left(2^{\dot{\alpha}}\left(\frac{5 \varkappa_{1}^{p}+\varkappa_{2}^{p}}{6}\right)^{\dot{\alpha}\left(1+\frac{1}{p}\right)}-\varkappa_{1}^{\dot{\alpha}(p+1)}-\left(\frac{\varkappa_{1}^{p}+\varkappa_{2}^{p}}{2}\right)^{\dot{\alpha}(1+1 / p)}\right)\right\}\right], \\
& \Lambda_{2}^{(\dot{\alpha})}\left(\varkappa_{1},\left(\frac{\varkappa_{1}^{p}+\varkappa_{2}^{p}}{2}\right)^{1 / p} ; p\right) \\
& =:\left(\frac{2}{3}\right)^{\dot{\alpha}}\left(\frac{p}{\left(\varkappa_{2}^{p}-\varkappa_{1}^{p}\right)^{2}}\right)^{\dot{\alpha}}\left[\frac { ( ( 5 \varkappa _ { 1 } ^ { p } + \varkappa _ { 2 } ^ { p } ) ( \varkappa _ { 1 } ^ { p } + \varkappa _ { 2 } ^ { p } ) ) ^ { \dot { \alpha } } } { \Gamma ( 1 + \dot { \alpha } ) } \left\{\left(\left(\frac{5 \varkappa_{1}^{p}+\varkappa_{2}^{p}}{6}\right)^{1 / p}-\varkappa_{1}\right)^{\dot{\alpha}}\right.\right. \\
& \left.-\left(\left(\frac{\varkappa_{1}^{p}+\varkappa_{2}^{p}}{2}\right)^{1 / p}-\left(\frac{5 \varkappa_{1}^{p}+\varkappa_{2}^{p}}{6}\right)^{1 / p}\right)^{\dot{\alpha}}\right\}-\frac{8^{\dot{\alpha}}\left(2 \varkappa_{1}^{p}+\varkappa_{2}^{p}\right) \Gamma(1+p \dot{\alpha})}{\Gamma(1+(p+1) \dot{\alpha})} \\
& \times\left\{2^{\dot{\alpha}}\left(\frac{5 \varkappa_{1}^{p}+\varkappa_{2}^{p}}{6}\right)^{\dot{\alpha}(1+1 / p)}-\left(\frac{\varkappa_{1}^{p}+\varkappa_{2}^{p}}{2}\right)^{\dot{\alpha}(1+1 / p)}-\varkappa_{1}^{\dot{\alpha}(p+1)}\right\}+\frac{\left(12^{\dot{\alpha}}\right) \Gamma(1+2 p \dot{\alpha})}{\Gamma(1+(2 p+1) \dot{\alpha})} \\
& \left.\times\left\{2^{\dot{\alpha}}\left(\frac{5 \varkappa_{1}^{p}+\varkappa_{2}^{p}}{6}\right)^{\dot{\alpha}(2+1 / p)}-\left(\frac{\varkappa_{1}^{p}+\varkappa_{2}^{p}}{2}\right)^{\dot{\alpha}(2+1 / p)}-\varkappa_{1}^{\dot{\alpha}(2 p+1)}\right\}\right] \text {, } \\
& \Lambda_{3}^{(\dot{\alpha})}\left(\varkappa_{1},\left(\frac{\varkappa_{1}^{p}+\varkappa_{2}^{p}}{2}\right)^{1 / p} ; p\right) \\
& =:\left(\frac{2}{3}\right)^{\dot{\alpha}}\left(\frac{p}{\left(\varkappa_{2}^{p}-\varkappa_{1}^{p}\right)^{2}}\right)^{\dot{\alpha}}\left[\frac { ( 1 1 \varkappa _ { 1 } ^ { p } + \varkappa _ { 2 } ^ { p } ) \Gamma ( 1 + p \dot { \alpha } ) } { \Gamma ( 1 + ( p + 1 ) \dot { \alpha } ) } \left\{2^{\dot{\alpha}}\left(\frac{5 \varkappa_{1}^{p}+\varkappa_{2}^{p}}{6}\right)^{\dot{\alpha}(1+1 / p)}\right.\right. \\
& \left.-\left(\frac{\varkappa_{1}^{p}+\varkappa_{2}^{p}}{2}\right)^{\dot{\alpha}(1+1 / p)}-\varkappa_{1}^{\dot{\alpha}(p+1)}\right\}-\frac{\left(6^{\dot{\alpha}}\right) \Gamma(1+2 p \dot{\alpha})}{\Gamma(1+(2 p+1) \dot{\alpha})}\left\{2^{\dot{\alpha}}\left(\frac{5 \varkappa_{1}^{p}+\varkappa_{2}^{p}}{6}\right)^{\dot{\alpha}(2+1 / p)}\right. \\
& \left.-\left(\frac{\varkappa_{1}^{p}+\varkappa_{2}^{p}}{2}\right)^{\dot{\alpha}(2+1 / p)}-\varkappa_{1}^{\dot{\alpha}(2 p+1)}\right\}-\frac{\left(\varkappa_{1}^{\dot{\alpha}}\left(5 \varkappa_{1}^{p}+\varkappa_{2}^{p}\right)\right.}{\Gamma(1+\dot{\alpha})}\left\{\left(\left(\frac{5 \varkappa_{1}^{p}+\varkappa_{2}^{p}}{6}\right)^{1 / p}-\varkappa_{1}\right)^{\dot{\alpha}}\right. \\
& \left.\left.-\left(\left(\frac{\varkappa_{1}^{p}+\varkappa_{2}^{p}}{2}\right)^{1 / p}-\left(\frac{5 \varkappa_{1}^{p}+\varkappa_{2}^{p}}{6}\right)^{1 / p}\right)^{\dot{\alpha}}\right\}\right] \text {, } \\
& \Lambda_{4}^{(\dot{\alpha})}\left(\left(\frac{\varkappa_{1}^{p}+\varkappa_{2}^{p}}{2}\right)^{1 / p}, \varkappa_{2} ; p\right) \\
& =:\left(\frac{2}{3}\right)^{\dot{\alpha}}\left(\frac{p}{\left(\varkappa_{2}^{p}-\varkappa_{1}^{p}\right)^{2}}\right)^{\dot{\alpha}}\left[\frac { ( \varkappa _ { 1 } ^ { p } + 5 \varkappa _ { 2 } ^ { p } ) ^ { \dot { \alpha } } } { \Gamma ( 1 + \dot { \alpha } ) } \left\{\left(\left(\frac{\varkappa_{1}^{p}+5 \varkappa_{2}^{p}}{6}\right)^{\frac{1}{p}}-\left(\frac{\varkappa_{1}^{p}+\varkappa_{2}^{p}}{2}\right)^{\frac{1}{p}}\right)^{\dot{\alpha}}\right.\right. \\
& \left.-\left(\varkappa_{2}-\left(\frac{\varkappa_{1}^{p}+5 \varkappa_{2}^{p}}{6}\right)^{1 / p}\right)^{\dot{\alpha}}\right\}-\frac{6^{\dot{\alpha}} \Gamma(1+p \dot{\alpha})}{\Gamma(1+(p+1) \dot{\alpha})} \\
& \left.\times\left\{2^{\dot{\alpha}}\left(\frac{\varkappa_{1}^{p}+5 \varkappa_{2}^{p}}{6}\right)^{\dot{\alpha}\left(1+\frac{1}{p}\right)}-\left(\frac{\varkappa_{1}^{p}+\varkappa_{2}^{p}}{2}\right)^{\dot{\alpha}\left(1+\frac{1}{p}\right)}-\varkappa_{2}^{\dot{\alpha}(p+1)}\right\}\right], \\
& \Lambda_{5}^{(\dot{\alpha})}\left(\left(\frac{\varkappa_{1}^{p}+\varkappa_{2}^{p}}{2}\right)^{1 / p}, \varkappa_{2} ; p\right) \\
& =:\left(\frac{2}{3}\right)^{\dot{\alpha}}\left(\frac{p}{\left(\varkappa_{2}^{p}-\varkappa_{1}^{p}\right)^{2}}\right)^{\dot{\alpha}}\left[\frac { 6 ^ { \dot { \alpha } } \Gamma ( 1 + 2 p \dot { \alpha } ) } { \Gamma ( 1 + ( 2 p + 1 ) \dot { \alpha } ) } \left\{2^{\dot{\alpha}}\left(\frac{\varkappa_{1}^{p}+5 \varkappa_{2}^{p}}{6}\right)^{\dot{\alpha}(2+1 / p)}-\varkappa_{2}^{\dot{\alpha}(2 p+1)}\right.\right. \\
& \left.-\left(\frac{\varkappa_{1}^{p}+\varkappa_{2}^{p}}{2}\right)^{\dot{\alpha}(2+1 / p)}\right\}-\frac{\left(\varkappa_{1}^{p}+11 \varkappa_{2}^{p}\right)^{\dot{\alpha}} \Gamma(1+p \dot{\alpha})}{\Gamma(1+(p+1) \dot{\alpha})}\left\{2^{\dot{\alpha}}\left(\frac{\varkappa_{1}^{p}+5 \varkappa_{2}^{p}}{6}\right)^{\dot{\alpha}(1+1 / p)}\right. \\
& \left.-\left(\frac{\varkappa_{1}^{p}+\varkappa_{2}^{p}}{2}\right)^{\dot{\alpha}(1+1 / p)}-\varkappa_{1}^{\dot{\alpha}(p+1)}\right\}-\frac{\left.\left(\varkappa_{2}^{p}\left(\varkappa_{1}^{p}+5 \varkappa_{2}^{p}\right)\right)\right)^{\dot{\alpha}}}{\Gamma(1+\dot{\alpha})} \\
& \left.\times\left\{\left(\left(\frac{\varkappa_{1}^{p}+5 \varkappa_{2}^{p}}{6}\right)^{1 / p}-\left(\frac{\varkappa_{1}^{p}+\varkappa_{2}^{p}}{2}\right)^{1 / p}\right)^{\dot{\alpha}}-\left(\left(\varkappa_{2}-\frac{\varkappa_{1}^{p}+5 \varkappa_{2}^{p}}{6}\right)^{1 / p}\right)^{\dot{\alpha}}\right\}\right],
\end{aligned}
$$


and

$$
\begin{aligned}
\Lambda_{6}^{(\dot{\alpha})} & \left(\left(\frac{\varkappa_{1}^{p}+\varkappa_{2}^{p}}{2}\right)^{1 / p}, \varkappa_{2} ; p\right) \\
= & \left(\frac{2}{3}\right)^{\dot{\alpha}}\left(\frac{p}{\left(\varkappa_{2}^{p}-\varkappa_{1}^{p}\right)^{2}}\right)^{\dot{\alpha}} \\
& \times\left[\frac{\left(\varkappa_{1}^{p}+\varkappa_{2}^{p}\right)^{\dot{\alpha}}\left(\varkappa_{1}^{p}+5 \varkappa_{2}^{p}\right)^{\dot{\alpha}}}{\Gamma(1+\dot{\alpha})}\left\{\left(\frac{\varkappa_{1}^{p}+5 \varkappa_{2}^{p}}{6}\right)^{1 / p}-\left(\frac{\varkappa_{1}^{p}+\varkappa_{2}^{p}}{2}\right)^{1 / p}\right)^{\dot{\alpha}}\right. \\
& \left.-\left(\varkappa_{2}-\left(\frac{\varkappa_{1}^{p}+5 \varkappa_{2}^{p}}{6}\right)^{1 / p}\right)^{\dot{\alpha}}\right\}+\frac{8^{\dot{\alpha}}\left(\varkappa_{1}^{p}+2 \varkappa_{2}^{p}\right)^{\dot{\alpha}} \Gamma(1+p \dot{\alpha})}{\Gamma(1+(p+1) \dot{\alpha})}\left\{2^{\dot{\alpha}}\left(\frac{\varkappa_{1}^{p}+5 \varkappa_{2}^{p}}{6}\right)^{\dot{\alpha}(1+1 / p)}\right. \\
& \left.-\left(\frac{\varkappa_{1}^{p}+\varkappa_{2}^{p}}{2}\right)^{\dot{\alpha}(1+1 / p)}-\varkappa_{2}^{\dot{\alpha}(p+1)}\right\}-\frac{(12)^{\dot{\alpha}} \Gamma(1+2 p \dot{\alpha})}{\Gamma(1+(2 p+1) \dot{\alpha})}\left\{2^{\dot{\alpha}}\left(\frac{\varkappa_{1}^{p}+5 \varkappa_{2}^{p}}{6}\right)^{\dot{\alpha}(2+1 / p)}\right. \\
& \left.\left.-\left(\frac{\varkappa_{1}^{p}+\varkappa_{2}^{p}}{2}\right)^{\dot{\alpha}(2+1 / p)}-\varkappa_{1}^{\dot{\alpha}(2 p+1)}\right\}\right] .
\end{aligned}
$$

Proof It follows from Lemma 3.4 and the generalized power mean inequality that

$$
\begin{aligned}
& \mid\left(\frac{1}{6}\right)^{\dot{\alpha}}\left[\chi\left(\varkappa_{1}\right)+(4)^{\dot{\alpha}} \chi\left(\left[\frac{\varkappa_{1}^{p}+\varkappa_{2}^{p}}{2}\right]^{1 / p}\right)+\chi\left(\varkappa_{2}\right)\right] \\
& -\left(\frac{2 p}{\varkappa_{2}^{p}-\varkappa_{1}^{p}}\right)^{\dot{\alpha}} \varkappa_{1} \mathcal{I}_{\varkappa_{2}(\dot{\alpha})} \frac{\chi(u)}{u^{\dot{\alpha}(1-p)}} \mid \\
& \leq\left(\frac{\varkappa_{2}^{p}-\varkappa_{1}^{p}}{4 p}\right)^{\dot{\alpha}}\left[\frac{1}{\Gamma(1+\dot{\alpha})} \int_{0}^{1} \frac{\left|\xi-\frac{1}{3}\right|^{\dot{\alpha}}}{\left[(1-\xi) \varkappa_{1}^{p}+\xi \frac{\varkappa_{1}^{p}+\varkappa_{2}^{p}}{2}\right]^{\dot{\alpha}(1-1 / p)}}\right. \\
& \times\left|\chi^{(\dot{\alpha})}\left(\left[(1-\xi) \varkappa_{1}^{p}+\xi \frac{\varkappa_{1}^{p}+\varkappa_{2}^{p}}{2}\right]^{1 / p}\right)\right|(d \xi)^{\dot{\alpha}} \\
& +\frac{1}{\Gamma(1+\dot{\alpha})} \int_{0}^{1} \frac{\left|\xi-\frac{2}{3}\right|^{\dot{\alpha}}}{\left[(1-\xi) \frac{\varkappa_{1}^{p}+\varkappa_{2}^{p}}{2}+\xi \varkappa_{2}^{p}\right]^{\dot{\alpha}(1-1 / p)}} \\
& \left.\times\left|\chi^{(\dot{\alpha})}\left(\left[(1-\xi) \frac{\varkappa_{1}^{p}+\varkappa_{2}^{p}}{2}+\xi \varkappa_{2}^{p}\right]^{1 / p}\right)\right|(d \xi)^{\dot{\alpha}}\right] \\
& \leq\left(\frac{\varkappa_{2}^{p}-\varkappa_{1}^{p}}{4 p}\right)^{\dot{\alpha}}\left[\left(\frac{1}{\Gamma(1+\dot{\alpha})} \int_{0}^{1} \frac{\left|\xi-\frac{1}{3}\right|^{\dot{\alpha}}}{\left[(1-\xi) \varkappa_{1}^{p}+\xi \frac{\varkappa_{1}^{p}+\varkappa_{2}^{p}}{2}\right]^{\dot{\alpha}(1-1 / p)}}(d \xi)^{\dot{\alpha}}\right)^{1-\frac{1}{q}}\right. \\
& \times\left(\frac{1}{\Gamma(1+\dot{\alpha})} \int_{0}^{1} \frac{\mid \xi-\frac{1}{3} \dot{\alpha}^{\dot{\alpha}}}{\left[(1-\xi) \varkappa_{1}^{p}+\xi \frac{\varkappa_{1}^{p}+\varkappa_{2}^{p}}{2}\right]^{\dot{\alpha}(1-1 / p)}}\right. \\
& \left.\times\left|\chi^{(\dot{\alpha})}\left(\left[(1-\xi) \varkappa_{1}^{p}+\xi \frac{\varkappa_{1}^{p}+\varkappa_{2}^{p}}{2}\right]^{1 / p}\right)\right|^{q}(d \xi)^{\dot{\alpha}}\right)^{\frac{1}{q}} \\
& +\left(\frac{1}{\Gamma(1+\dot{\alpha})} \int_{0}^{1} \frac{\left|\xi-\frac{2}{3}\right|^{\dot{\alpha}}}{\left[(1-\xi) \frac{\varkappa_{1}^{p}+\varkappa_{2}^{p}}{2}+\xi \varkappa_{2}^{p}\right]^{\dot{\alpha}(1-1 / p)}}(d \xi)^{\dot{\alpha}}\right)^{1-\frac{1}{q}} \\
& \times\left(\frac{1}{\Gamma(1+\dot{\alpha})} \int_{0}^{1} \frac{\left|\xi-\frac{2}{3}\right|^{\dot{\alpha}}}{\left[(1-\xi) \frac{\varkappa_{1}^{p}+\varkappa_{2}^{p}}{2}+\xi \varkappa_{2}^{p}\right]^{\dot{\alpha}(1-1 / p)}}\right. \\
& \left.\left.\times\left|\chi^{(\dot{\alpha})}\left(\left[(1-\xi) \frac{\varkappa_{1}^{p}+\varkappa_{2}^{p}}{2}+\xi \varkappa_{2}^{p}\right]^{1 / p}\right)\right|^{q}(d \xi)^{\dot{\alpha}}\right)^{\frac{1}{q}}\right] .
\end{aligned}
$$


Making use of the generalized $p$-convexity of $\left|\chi^{(\dot{\alpha})}\right|^{q}$ on $\Omega$, we have

$$
\begin{aligned}
& \mid\left(\frac{1}{6}\right)^{\dot{\alpha}}\left[\chi\left(\varkappa_{1}\right)+(4)^{\dot{\alpha}} \chi\left(\left[\frac{\varkappa_{1}^{p}+\varkappa_{2}^{p}}{2}\right]^{1 / p}\right)+\chi\left(\varkappa_{2}\right)\right] \\
& -\left(\frac{2 p}{\varkappa_{2}^{p}-\varkappa_{1}^{p}}\right)^{\dot{\alpha}} \varkappa_{1} \mathcal{I}_{\varkappa_{2}}^{(\dot{\alpha})} \frac{\chi(u)}{u^{\dot{\alpha}(1-p)}} \\
& \leq\left(\frac{\varkappa_{2}^{p}-\varkappa_{1}^{p}}{4 p}\right)^{\dot{\alpha}}\left[\left(\frac{1}{\Gamma(1+\dot{\alpha})} \int_{0}^{1} \frac{\left|\xi-\frac{1}{3}\right|^{\dot{\alpha}}}{\left[(1-\xi) \varkappa_{1}^{p}+\xi \frac{\varkappa_{1}^{p}+\varkappa_{2}^{p}}{2}\right]^{\dot{\alpha}(1-1 / p)}}(d \xi)^{\dot{\alpha}}\right)^{1-\frac{1}{q}}\right. \\
& \times\left(\frac{1}{\Gamma(1+\dot{\alpha})} \int_{0}^{1} \frac{\left|\xi-\frac{1}{3}\right|^{\dot{\alpha}}}{\left[(1-\xi) \varkappa_{1}^{p}+\xi \frac{\varkappa_{1}^{p}+\varkappa_{2}^{p}}{2}\right]^{\dot{\alpha}(1-1 / p)}}\right. \\
& \left.\times\left[(1-\xi)^{\dot{\alpha}}\left|\chi^{(\dot{\alpha})}\left(\varkappa_{1}\right)\right|^{q}+\xi^{\dot{\alpha}}\left|\chi^{(\dot{\alpha})}\left(\frac{\varkappa_{1}^{p}+\varkappa_{2}^{p}}{2}\right)^{1 / p}\right|^{q}\right](d \xi)^{\dot{\alpha}}\right)^{\frac{1}{q}} \\
& +\left(\frac{1}{\Gamma(1+\dot{\alpha})} \int_{0}^{1} \frac{\left|\xi-\frac{2}{3}\right|^{\dot{\alpha}}}{\left[(1-\xi) \frac{\varkappa_{1}^{p}+\varkappa_{2}^{p}}{2}+\xi \varkappa_{2}^{p}\right]^{\dot{\alpha}(1-1 / p)}}(d \xi)^{\dot{\alpha}}\right)^{1-\frac{1}{q}} \\
& \times\left(\frac{1}{\Gamma(1+\dot{\alpha})} \int_{0}^{1} \frac{\left|\xi-\frac{2}{3}\right|^{\dot{\alpha}}}{\left[(1-\xi) \frac{\varkappa_{1}^{p}+\varkappa_{2}^{p}}{2}+\xi \varkappa_{2}^{p}\right]^{\dot{\alpha}(1-1 / p)}}\right. \\
& \left.\left.\times\left[(1-\xi)^{\dot{\alpha}}\left|\chi^{(\dot{\alpha})}\left(\frac{\varkappa_{1}^{p}+\varkappa_{2}^{p}}{2}\right)^{1 / p}\right|^{q}+\xi^{\dot{\alpha}}\left|\chi^{(\dot{\alpha})}\left(\varkappa_{2}\right)\right|^{q}\right](d \xi)^{\dot{\alpha}}\right)^{\frac{1}{q}}\right] \\
& =\left(\frac{\varkappa_{2}^{p}-\varkappa_{1}^{p}}{4 p}\right)^{\dot{\alpha}}\left[\left(\Lambda_{1}^{(\dot{\alpha})}\left(\varkappa_{1},\left(\frac{\varkappa_{1}^{p}+\varkappa_{2}^{p}}{2}\right)^{1 / p} ; p\right)\right)^{1-\frac{1}{q}}\right. \\
& \times\left[\Lambda_{2}^{(\dot{\alpha})}\left(\varkappa_{1},\left(\frac{\varkappa_{1}^{p}+\varkappa_{2}^{p}}{2}\right)^{1 / p} ; p\right)\left|\chi^{(\dot{\alpha})}\left(\varkappa_{1}\right)\right|^{q}\right. \\
& \left.+\Lambda_{3}^{(\dot{\alpha})}\left(\varkappa_{1},\left(\frac{\varkappa_{1}^{p}+\varkappa_{2}^{p}}{2}\right)^{1 / p} ; p\right)\left|\chi^{(\dot{\alpha})}\left(\frac{\varkappa_{1}^{p}+\varkappa_{2}^{p}}{2}\right)^{1 / p}\right|^{q}\right]^{\frac{1}{q}} \\
& +\left(\Lambda_{4}^{(\dot{\alpha})}\left(\left(\frac{\varkappa_{1}^{p}+\varkappa_{2}^{p}}{2}\right)^{1 / p}, \varkappa_{2} ; p\right)\right)^{1-\frac{1}{q}} \\
& \times\left[\Lambda_{5}^{(\dot{\alpha})}\left(\left(\frac{\varkappa_{1}^{p}+\varkappa_{2}^{p}}{2}\right)^{1 / p}, \varkappa_{2} ; p\right)\left|\chi^{(\dot{\alpha})}\left(\frac{\varkappa_{1}^{p}+\varkappa_{2}^{p}}{2}\right)^{1 / p}\right|^{q}\right. \\
& \left.\left.+\Lambda_{6}^{(\dot{\alpha})}\left(\left(\frac{\varkappa_{1}^{p}+\varkappa_{2}^{p}}{2}\right)^{1 / p}, \varkappa_{2} ; p\right)\left|\chi^{(\dot{\alpha})}\left(\varkappa_{2}\right)\right|^{q}\right]^{\frac{1}{q}}\right] \text {. }
\end{aligned}
$$

We use Lemma 2.5 and the facts that

$$
\begin{aligned}
& \Lambda_{1}^{(\dot{\alpha})}\left(\varkappa_{1},\left(\frac{\varkappa_{1}^{p}+\varkappa_{2}^{p}}{2}\right)^{1 / p} ; p\right) \\
& =: \frac{1}{\Gamma(1+\dot{\alpha})} \int_{0}^{1} \frac{\left|\xi-\frac{1}{3}\right|^{\dot{\alpha}}}{\left[(1-\xi) \varkappa_{1}^{p}+\xi \frac{\varkappa_{1}^{p}+\varkappa_{2}^{p}}{2}\right]^{\dot{\alpha}(1-1 / p)}}(d \xi)^{\dot{\alpha}} \\
& =\frac{1}{\Gamma(1+\dot{\alpha})} \int_{0}^{\frac{1}{3}} \frac{\left(\frac{1}{3}-\xi\right)^{\dot{\alpha}}}{\left[(1-\xi) \varkappa_{1}^{p}+\xi \frac{\varkappa_{1}^{p}+\varkappa_{2}^{p}}{2}\right]^{\dot{\alpha}(1-1 / p)}}(d \xi)^{\dot{\alpha}}
\end{aligned}
$$


Abdeljawad et al. Advances in Difference Equations

(2020) 2020:496

Page 11 of 26

$$
\begin{aligned}
& +\frac{1}{\Gamma(1+\dot{\alpha})} \int_{\frac{1}{3}}^{1} \frac{\left(\xi-\frac{1}{3}\right)^{\dot{\alpha}}}{\left[(1-\xi) \varkappa_{1}^{p}+\xi \frac{\varkappa_{1}^{p}+\varkappa_{2}^{p}}{2}\right]^{\dot{\alpha}(1-1 / p)}}(d \xi)^{\dot{\alpha}} \\
& =\left(\frac{2}{3}\right)^{\dot{\alpha}}\left(\frac{p}{\left(\varkappa_{2}^{p}-\varkappa_{1}^{p}\right)^{2}}\right)^{\dot{\alpha}}\left[\int_{\varkappa_{1}}^{\left(\frac{5 \varkappa_{1}^{p} \varkappa_{2}^{p}}{6}\right)^{1 / p}}\left[\left(\varkappa_{1}^{p}+\varkappa_{2}^{p}\right)^{\dot{\alpha}}-6^{\dot{\alpha}} u^{\dot{\alpha} p}\right](d u)^{\dot{\alpha}}\right. \\
& \left.\left.+\int_{\left(\frac{5 \varkappa_{1}^{p}+\varkappa_{2}^{p}}{6}\right)^{1 / p}}^{\left(\frac { \varkappa _ { 1 } ^ { p } + \varkappa _ { 2 } ^ { p } } { 2 } \left[6^{1 / p}\right.\right.} 6^{\dot{\alpha}} u^{\dot{\alpha} p}-\left(\varkappa_{1}^{p}+\varkappa_{2}^{p}\right)^{\dot{\alpha}}\right](d u)^{\dot{\alpha}}\right] \\
& =\left(\frac{2}{3}\right)^{\dot{\alpha}}\left(\frac{p}{\left(\varkappa_{2}^{p}-\varkappa_{1}^{p}\right)^{2}}\right)^{\dot{\alpha}}\left[\frac { ( \varkappa _ { 1 } ^ { p } + \varkappa _ { 2 } ^ { p } ) ^ { \dot { \alpha } } } { \Gamma ( 1 + \dot { \alpha } ) } \left\{\left(\left(\frac{5 \varkappa_{1}^{p}+\varkappa_{2}^{p}}{6}\right)^{\frac{1}{p}}-\varkappa_{1}\right)^{\dot{\alpha}}\right.\right. \\
& \left.-\left(\left(\frac{\varkappa_{1}^{p}+\varkappa_{2}^{p}}{2}\right)^{1 / p}-\left(\frac{5 \varkappa_{1}^{p}+\varkappa_{2}^{p}}{6}\right)^{\frac{1}{p}}\right)^{\dot{\alpha}}\right\} \\
& -\frac{6^{\dot{\alpha}} \Gamma(1+p \dot{\alpha})}{\Gamma(1+(p+1) \dot{\alpha})}\left\{\left(\left(\frac{5 \varkappa_{1}^{p}+\varkappa_{2}^{p}}{6}\right)^{\dot{\alpha}\left(1+\frac{1}{p}\right)}-\varkappa_{1}^{\dot{\alpha}(p+1)}\right)\right. \\
& \left.\left.-\left(\left(\frac{\varkappa_{1}^{p}+\varkappa_{2}^{p}}{2}\right)^{\dot{\alpha}(1+1 / p)}-\left(\frac{5 \varkappa_{1}^{p}+\varkappa_{2}^{p}}{6}\right)^{\dot{\alpha}\left(1+\frac{1}{p}\right)}\right)\right\}\right], \\
& \Lambda_{2}^{(\dot{\alpha})}\left(\varkappa_{1},\left(\frac{\varkappa_{1}^{p}+\varkappa_{2}^{p}}{2}\right)^{1 / p} ; p\right) \\
& =: \frac{1}{\Gamma(1+\dot{\alpha})} \int_{0}^{1} \frac{\left|\xi-\frac{1}{3}\right|^{\dot{\alpha}}(1-\xi)^{\dot{\alpha}}(d \xi)^{\dot{\alpha}}}{\left[(1-\xi) \varkappa_{1}^{p}+\xi \frac{\varkappa_{1}^{p}+\varkappa_{2}^{p}}{2}\right]^{\dot{\alpha}(1-1 / p)}} \\
& =\frac{1}{\Gamma(1+\dot{\alpha})} \int_{0}^{1 / 3} \frac{\left(\frac{1}{3}-\xi\right)^{\dot{\alpha}}(1-\xi)^{\dot{\alpha}}(d \xi)^{\dot{\alpha}}}{\left[\left[(1-\xi) \varkappa_{1}^{p}+\xi \frac{\varkappa_{1}^{p}+\varkappa_{2}^{p}}{2}\right]^{\dot{\alpha}(1-1 / p)}\right.} \\
& +\frac{1}{\Gamma(1+\dot{\alpha})} \int_{1 / 3}^{1} \frac{\left(\xi-\frac{1}{3}\right)^{\dot{\alpha}}(1-\xi)^{\dot{\alpha}}(d \xi)^{\dot{\alpha}}}{\left[\left[(1-\xi) \varkappa_{1}^{p}+\xi \frac{\varkappa_{1}^{p}+\varkappa_{2}^{p}}{2}\right]^{\dot{\alpha}(1-1 / p)}\right.} \\
& =\left(\frac{2}{3}\right)^{\dot{\alpha}}\left(\frac{p}{\left(\varkappa_{2}^{p}-\varkappa_{1}^{p}\right)^{2}}\right)^{\dot{\alpha}}\left[\frac { ( ( 5 \varkappa _ { 1 } ^ { p } + \varkappa _ { 2 } ^ { p } ) ( \varkappa _ { 1 } ^ { p } + \varkappa _ { 2 } ^ { p } ) ) ^ { \dot { \alpha } } } { \Gamma ( 1 + \dot { \alpha } ) } \left\{\left(\left(\frac{5 \varkappa_{1}^{p}+\varkappa_{2}^{p}}{6}\right)^{1 / p}-\varkappa_{1}\right)^{\dot{\alpha}}\right.\right. \\
& \left.-\left(\left(\frac{\varkappa_{1}^{p}+\varkappa_{2}^{p}}{2}\right)^{1 / p}-\left(\frac{5 \varkappa_{1}^{p}+\varkappa_{2}^{p}}{6}\right)^{1 / p}\right)^{\dot{\alpha}}\right\}-\frac{8^{\dot{\alpha}}\left(2 \varkappa_{1}^{p}+\varkappa_{2}^{p}\right) \Gamma(1+p \dot{\alpha})}{\Gamma(1+(p+1) \dot{\alpha})} \\
& \times\left\{2^{\dot{\alpha}}\left(\frac{5 \varkappa_{1}^{p}+\varkappa_{2}^{p}}{6}\right)^{\dot{\alpha}(1+1 / p)}-\left(\frac{\varkappa_{1}^{p}+\varkappa_{2}^{p}}{2}\right)^{\dot{\alpha}(1+1 / p)}-\varkappa_{1}^{\dot{\alpha}(p+1)}\right\} \\
& \left.+\frac{\left(12^{\dot{\alpha}}\right) \Gamma(1+2 p \dot{\alpha})}{\Gamma(1+(2 p+1) \dot{\alpha})}\left\{2^{\dot{\alpha}}\left(\frac{5 \varkappa_{1}^{p}+\varkappa_{2}^{p}}{6}\right)^{\dot{\alpha}(2+1 / p)}-\left(\frac{\varkappa_{1}^{p}+\varkappa_{2}^{p}}{2}\right)^{\dot{\alpha}(2+1 / p)}-\varkappa_{1}^{\dot{\alpha}(2 p+1)}\right\}\right],
\end{aligned}
$$

and

$$
\begin{aligned}
& \Lambda_{3}^{(\dot{\alpha})}\left(\varkappa_{1},\left(\frac{\varkappa_{1}^{p}+\varkappa_{2}^{p}}{2}\right)^{1 / p} ; p\right) \\
& =: \frac{1}{\Gamma(1+\dot{\alpha})} \int_{0}^{1} \frac{\left|\xi-\frac{1}{3}\right|^{\dot{\alpha}} \xi^{\dot{\alpha}}(d \xi)^{\dot{\alpha}}}{\left[(1-\xi) \varkappa_{1}^{p}+\xi \frac{\varkappa_{1}^{p}+\varkappa_{2}^{p}}{2}\right]^{\dot{\alpha}(1-1 / p)}}
\end{aligned}
$$




$$
\begin{aligned}
= & \frac{1}{\Gamma(1+\dot{\alpha})} \int_{0}^{1 / 3} \frac{\left(\frac{1}{3}-\xi\right)^{\dot{\alpha}} \xi^{\dot{\alpha}}(d \xi)^{\dot{\alpha}}}{\left[(1-\xi) \varkappa_{1}^{p}+\xi \frac{\varkappa_{1}^{p}+\varkappa_{2}^{p}}{2}\right]^{\dot{\alpha}(1-1 / p)}} \\
& +\frac{1}{\Gamma(1+\dot{\alpha})} \int_{1 / 3}^{1} \frac{\left(\xi-\frac{1}{3}\right)^{\dot{\alpha}} \xi^{\dot{\alpha}}(d \xi)^{\dot{\alpha}}}{\left[(1-\xi) \varkappa_{1}^{p}+\xi \frac{\varkappa_{1}^{p}+\varkappa_{2}^{p}}{2}\right]^{\dot{\alpha}(1-1 / p)}} \\
= & \left(\frac{2}{3}\right)^{\dot{\alpha}}\left(\frac{p}{\left(\varkappa_{2}^{p}-\varkappa_{1}^{p}\right)^{2}}\right)^{\dot{\alpha}}\left[\frac { ( 1 1 \varkappa _ { 1 } ^ { p } + \varkappa _ { 2 } ^ { p } ) \Gamma ( 1 + p \dot { \alpha } ) } { \Gamma ( 1 + ( p + 1 ) \dot { \alpha } ) } \left\{2^{\dot{\alpha}}\left(\frac{5 \varkappa_{1}^{p}+\varkappa_{2}^{p}}{6}\right)^{\dot{\alpha}(1+1 / p)}\right.\right. \\
& \left.-\left(\frac{\varkappa_{1}^{p}+\varkappa_{2}^{p}}{2}\right)^{\dot{\alpha}(1+1 / p)}-\varkappa_{1}^{\dot{\alpha}(p+1)}\right\}-\frac{\left(6^{\dot{\alpha}}\right) \Gamma(1+2 p \dot{\alpha})}{\Gamma(1+(2 p+1) \dot{\alpha})}\left\{2^{\dot{\alpha}}\left(\frac{5 \varkappa_{1}^{p}+\varkappa_{2}^{p}}{6}\right)^{\dot{\alpha}(2+1 / p)}\right. \\
& \left.-\left(\frac{\varkappa_{1}^{p}+\varkappa_{2}^{p}}{2}\right)^{\dot{\alpha}(2+1 / p)}-\varkappa_{1}^{\dot{\alpha}(2 p+1)}\right\}-\frac{\left(\varkappa_{1}^{\dot{\alpha}}\left(5 \varkappa_{1}^{p}+\varkappa_{2}^{p}\right)\right.}{\Gamma(1+\dot{\alpha})} \\
& \left.\times\left\{\left(\left(\frac{5 \varkappa_{1}^{p}+\varkappa_{2}^{p}}{6}\right)^{1 / p}-\varkappa_{1}\right)^{\dot{\alpha}}-\left(\left(\frac{\varkappa_{1}^{p}+\varkappa_{2}^{p}}{2}\right)^{1 / p}-\left(\frac{5 \varkappa_{1}^{p}+\varkappa_{2}^{p}}{6}\right)^{1 / p}\right)^{\dot{\alpha}}\right\}\right],
\end{aligned}
$$

where we have used the identities

$$
\begin{aligned}
& \Lambda_{4}^{(\dot{\alpha})}\left(\left(\frac{\varkappa_{1}^{p}+\varkappa_{2}^{p}}{2}\right)^{1 / p}, \varkappa_{2} ; p\right):=\frac{1}{\Gamma(1+\dot{\alpha})} \int_{0}^{1} \frac{\left|\xi-\frac{2}{3}\right|^{\dot{\alpha}}}{\left[(1-\xi) \frac{\varkappa_{1}^{p}+\varkappa_{2}^{p}}{2}+\xi \varkappa_{2}^{p}\right]^{\dot{\alpha}(1-1 / p)}}(d \xi)^{\dot{\alpha}}, \\
& \Lambda_{5}^{(\dot{\alpha})}\left(\left(\frac{\varkappa_{1}^{p}+\varkappa_{2}^{p}}{2}\right)^{1 / p}, \varkappa_{2} ; p\right):=\frac{1}{\Gamma(1+\dot{\alpha})} \int_{0}^{1} \frac{\left|\xi-\frac{2}{3}\right|^{\dot{\alpha}}(1-\xi)^{\dot{\alpha}}}{\left[(1-\xi) \frac{\varkappa_{1}^{p}+\varkappa_{2}^{p}}{2}+\xi \varkappa_{2}^{p}\right]^{\dot{\alpha}(1-1 / p)}}(d \xi)^{\dot{\alpha}}, \\
& \Lambda_{6}^{(\dot{\alpha})}\left(\left(\frac{\varkappa_{1}^{p}+\varkappa_{2}^{p}}{2}\right)^{1 / p}, \varkappa_{2} ; p\right):=\frac{1}{\Gamma(1+\dot{\alpha})} \int_{0}^{1} \frac{\left|\xi-\frac{2}{3}\right|^{\dot{\alpha}} \xi^{\dot{\alpha}}}{\left[(1-\xi) \frac{\varkappa_{1}^{p}+\varkappa_{2}^{p}}{2}+\xi \varkappa_{2}^{p}\right]^{\dot{\alpha}(1-1 / p)}}(d \xi)^{\dot{\alpha}} .
\end{aligned}
$$

Substituting (3.14)-(3.16) in (3.12), we conclude the immediate consequence (3.5). This completes the proof.

Remark 3.6 Theorem 3.5 leads to the following conclusions:

(1) Let $\dot{\alpha}=1$. Then we get Theorem 2.1 of [29].

(2) Let $\dot{\alpha}=p=1$. Then we get Corollary 10 of [71] if we choose $s=1$, which also coincides with the first part of Corollary 2.1 of [29].

(3) Let $\dot{\alpha}=1$ and $p=-1$. Then we get the second part of Corollary 2.1 of [29].

Theorem 3.7 Let $p \in \mathbb{R} \backslash\{0\}, s, q>1$ with $s^{-1}+q^{-1}=1$ and $\chi: \Omega^{\circ} \subset \mathbb{R} \rightarrow \mathbb{R}^{\dot{\alpha}}$ such that $\chi \in \mathcal{D}_{\dot{\alpha}}\left(\Omega^{\circ}\right)\left(\Omega^{\circ}\right.$ is the interior of $\left.\Omega\right), \chi^{(\dot{\alpha})} \in \mathcal{C}_{\dot{\alpha}}\left[\varkappa_{1}, \varkappa_{2}\right]$ and $\left|\chi^{(\dot{\alpha})}\right|^{q}$ is generalized $p$-convex on $\Omega$. Then

$$
\begin{aligned}
& \left|\left(\frac{1}{6}\right)^{\dot{\alpha}}\left[\chi\left(\varkappa_{1}\right)+(4)^{\dot{\alpha}} \chi\left(\left[\frac{\varkappa_{1}^{p}+\varkappa_{2}^{p}}{2}\right]^{1 / p}\right)+\chi\left(\varkappa_{2}\right)\right]-\left(\frac{2 p}{\varkappa_{2}^{p}-\varkappa_{1}^{p}}\right)^{\dot{\alpha}} \varkappa_{1} \mathcal{I}_{\varkappa_{2}}^{(\dot{\alpha})} \frac{\chi(u)}{u^{\dot{\alpha}(1-p)}}\right| \\
& \leq\left(\frac{\varkappa_{2}^{p}-\varkappa_{1}^{p}}{4 p}\right)^{\dot{\alpha}}\left[\left(\Lambda_{7}^{(\dot{\alpha})}\left(\varkappa_{1},\left(\frac{\varkappa_{1}^{p}+\varkappa_{2}^{p}}{2}\right)^{1 / p} ; s, p\right)\right)^{\frac{1}{s}}\right. \\
& \quad \times\left[\frac{2^{\dot{\alpha}} \Gamma(1+\dot{\alpha})}{\Gamma(1+2 \dot{\alpha})} \mathbb{A}^{(\dot{\alpha})}\left(\left|\chi^{(\dot{\alpha})}\left(\varkappa_{1}\right)\right|^{q},\left|\chi^{(\dot{\alpha})}\left(\frac{\varkappa_{1}^{p}+\varkappa_{2}^{p}}{2}\right)^{1 / p}\right|^{q}\right)\right]^{\frac{1}{q}}
\end{aligned}
$$




$$
\begin{aligned}
& +\left(\Lambda_{8}^{(\dot{\alpha})}\left(\left(\frac{\varkappa_{1}^{p}+\varkappa_{2}^{p}}{2}\right)^{1 / p}, \varkappa_{2} ; s, p\right)\right)^{\frac{1}{s}} \\
& \left.\times\left[\frac{2^{\dot{\alpha}} \Gamma(1+\dot{\alpha})}{\Gamma(1+2 \dot{\alpha})} \mathbb{A}^{(\dot{\alpha})}\left(\left|\chi^{(\dot{\alpha})}\left(\frac{\varkappa_{1}^{p}+\varkappa_{2}^{p}}{2}\right)^{1 / p}\right|^{q},\left|\chi^{(\dot{\alpha})}\left(\varkappa_{2}\right)\right|^{q}\right)\right]^{\frac{1}{q}}\right],
\end{aligned}
$$

where

$$
\begin{aligned}
\Lambda_{7}^{(\dot{\alpha})} & \left(\varkappa_{1},\left(\frac{\varkappa_{1}^{p}+\varkappa_{2}^{p}}{2}\right)^{1 / p} ; s, p\right) \\
:= & 6^{\dot{\alpha}}\left\{\frac{\left(\left(\frac{5 \varkappa_{1}^{p}+\varkappa_{2}^{p}}{6}\right)^{(2-s)+s / p}-\varkappa_{1}^{p(2-s)+s}\right)^{\dot{\alpha}}}{(p(2-s)+s)^{\dot{\alpha}} \Gamma(1+\dot{\alpha})}\right. \\
& \left.+\frac{\left(\left(\frac{\varkappa_{1}^{p}+\varkappa_{2}^{p}}{2}\right)^{(2-s)+s / p}-\left(\frac{5 \varkappa_{1}^{p}+\varkappa_{2}^{p}}{6}\right)^{(2-s)+s / p}\right)^{\dot{\alpha}}}{(p(2-s)+s)^{\dot{\alpha}} \Gamma(1+\dot{\alpha})}\right\} \\
& -\left(5 \varkappa_{1}^{p}+\varkappa^{p}\right)^{s \dot{\alpha}}\left\{\frac{\left.\left(\frac{5 \varkappa_{1}^{p}+\varkappa_{2}^{p}}{6}\right)^{(1-s)+s / p}-\varkappa_{1}^{p(1-s)+s}\right)^{\dot{\alpha}}}{(p(1-s)+s)^{\dot{\alpha}} \Gamma(1+\dot{\alpha})}\right. \\
& +\frac{\left.\left(\left(\frac{\varkappa_{1}^{p}+\varkappa_{2}^{p}}{2}\right)^{(1-s)+s / p}-\left(\frac{5 \varkappa_{1}^{p}+\varkappa_{2}^{p}}{6}\right)^{(1-s)+s / p}\right)^{\dot{\alpha}}\right]}{(p(1-s)+s)^{\dot{\alpha}} \Gamma(1+\dot{\alpha})}, \\
\Lambda_{8}^{(\dot{\alpha})} & \left(\left(\frac{\varkappa_{1}^{p}+\varkappa_{2}^{p}}{2}\right)^{1 / p}, \varkappa_{2} ; s, p\right) \\
:= & \left(\varkappa_{1}^{p}+5 \varkappa^{p}\right)^{s \dot{\alpha}}\left\{\frac{\left.\left(\frac{\varkappa_{1}^{p}+5 \varkappa_{2}^{p}}{6}\right)^{(1-s)+s / p}-\left(\frac{\varkappa_{1}^{p}+\varkappa_{2}^{p}}{2}\right)^{(1-s)+s / p}\right)^{\dot{\alpha}}}{(p(1-s)+s)^{\dot{\alpha}} \Gamma(1+\dot{\alpha})}\right. \\
& +\frac{\left.\varkappa_{2}^{p(1-s)+s}-\left(\frac{\varkappa_{1}^{p}+5 \varkappa_{2}^{p}}{6}\right)^{(1-s)+s / p}\right)^{\dot{\alpha}}}{(p(1-s)+s)^{\dot{\alpha}} \Gamma(1+\dot{\alpha})} \\
& -6^{s \dot{\alpha}}\left\{\frac{\left.\left[\left(\frac{\varkappa_{1}^{p}+5 \varkappa_{2}^{p}}{6}\right)^{(2-s)+s / p}-\left(\frac{\varkappa_{1}^{p}+\varkappa_{2}^{p}}{2}\right)^{(2-s)+s / p}\right)^{\dot{\alpha}}\right)^{\dot{\alpha}}}{(p(2-s)+s)^{\dot{\alpha}} \Gamma(1+\dot{\alpha})}\right. \\
& +\frac{\left.\left(\varkappa_{2}^{p(2-s)+s}-\left(\frac{\varkappa_{1}^{p}+5 \varkappa_{2}^{p}}{6}\right)^{(2-s)+s / p}\right)^{\dot{\alpha}}\right]}{(p(2-s)+s)^{\dot{\alpha}} \Gamma(1+\dot{\alpha})},
\end{aligned}
$$

and $\mathbb{A}=\frac{\varkappa_{1}+\varkappa_{2}}{2}$ denotes the arithmetic mean.

Proof It follows from Lemma 3.4 and the generalized Hölder inequality that

$$
\begin{aligned}
& \mid\left(\frac{1}{6}\right)^{\dot{\alpha}}\left[\chi\left(\varkappa_{1}\right)+(4)^{\dot{\alpha}} \chi\left(\left[\frac{\varkappa_{1}^{p}+\varkappa_{2}^{p}}{2}\right]^{1 / p}\right)+\chi\left(\varkappa_{2}\right)\right] \\
& \quad-\left(\frac{2 p}{\varkappa_{2}^{p}-\varkappa_{1}^{p}}\right)^{\dot{\alpha}} \varkappa_{1} \mathcal{I}_{\varkappa_{2}}^{(\dot{\alpha})} \frac{\chi(u)}{u^{\dot{\alpha}(1-p)}} \mid \\
& \leq\left(\frac{\varkappa_{2}^{p}-\varkappa_{1}^{p}}{4 p}\right)^{\dot{\alpha}}\left[\left(\frac{1}{\Gamma(1+\dot{\alpha})} \int_{0}^{1} \frac{\left|\xi-\frac{1}{3}\right|^{s \dot{\alpha}}}{\left[(1-\xi) \varkappa_{1}^{p}+\xi \frac{\varkappa_{1}^{p}+\varkappa_{2}^{p}}{2}\right]^{s \dot{\alpha}(1-1 / p)}}(d \xi)^{\dot{\alpha}}\right)^{\frac{1}{s}}\right. \\
& \quad \times\left(\frac{1}{\Gamma(1+\dot{\alpha})} \int_{0}^{1}\left|\chi^{(\dot{\alpha})}\left(\left[(1-\xi) \varkappa_{1}^{p}+\xi \frac{\varkappa_{1}^{p}+\varkappa_{2}^{p}}{2}\right]^{1 / p}\right)\right|^{q}(d \xi)^{\dot{\alpha}}\right)^{\frac{1}{q}}
\end{aligned}
$$




$$
\begin{aligned}
& +\left(\frac{1}{\Gamma(1+\dot{\alpha})} \int_{0}^{1} \frac{\left(\xi-\frac{2}{3}\right)^{s \dot{\alpha}}}{\left[(1-\xi) \frac{\varkappa_{1}^{p}+\varkappa_{2}^{p}}{2}+\xi \varkappa_{2}^{p}\right]^{s \dot{\alpha}(1-1 / p)}}(d \xi)^{\dot{\alpha}}\right)^{\frac{1}{s}} \\
& \left.\times\left(\frac{1}{\Gamma(1+\dot{\alpha})} \int_{0}^{1}\left|\chi^{(\dot{\alpha})}\left(\left[(1-\xi) \frac{\varkappa_{1}^{p}+\varkappa_{2}^{p}}{2}+\xi \varkappa_{2}^{p}\right]^{1 / p}\right)\right|^{q}(d \xi)^{\dot{\alpha}}\right)^{\frac{1}{q}}\right] .
\end{aligned}
$$

From the generalized $p$-convexity of $\left|\chi^{(\dot{\alpha})}\right|^{q}$ on $\Omega$, we have

$$
\begin{aligned}
& \mid\left(\frac{1}{6}\right)^{\dot{\alpha}}\left[\chi\left(\varkappa_{1}\right)+(4)^{\dot{\alpha}} \chi\left(\left[\frac{\varkappa_{1}^{p}+\varkappa_{2}^{p}}{2}\right]^{1 / p}\right)+\chi\left(\varkappa_{2}\right)\right] \\
& -\left(\frac{2 p}{\varkappa_{2}^{p}-\varkappa_{1}^{p}}\right)^{\dot{\alpha}} \varkappa_{1} \mathcal{I}_{\varkappa_{2}}^{(\dot{\alpha})} \frac{\chi(u)}{u^{\dot{\alpha}(1-p)}} \\
& \leq\left(\frac{\varkappa_{2}^{p}-\varkappa_{1}^{p}}{4 p}\right)^{\dot{\alpha}}\left[\left(\frac{1}{\Gamma(1+\dot{\alpha})} \int_{0}^{1} \frac{\left|\xi-\frac{1}{3}\right|^{s \dot{\alpha}}}{\left[(1-\xi) \varkappa_{1}^{p}+\xi \frac{\varkappa_{1}^{p}+\varkappa_{2}^{p}}{2}\right]^{s \dot{\alpha}(1-1 / p)}}(d \xi)^{\dot{\alpha}}\right)^{\frac{1}{s}}\right. \\
& \times\left(\frac{1}{\Gamma(1+\dot{\alpha})} \int_{0}^{1}\left[(1-\xi)^{\dot{\alpha}}\left|\chi^{(\dot{\alpha})}\left(\varkappa_{1}\right)\right|^{q}+\xi^{\dot{\alpha}}\left|\chi^{(\dot{\alpha})}\left(\frac{\varkappa_{1}^{p}+\varkappa_{2}^{p}}{2}\right)^{1 / p}\right|^{q}\right](d \xi)^{\dot{\alpha}}\right)^{\frac{1}{q}} \\
& +\left(\frac{1}{\Gamma(1+\dot{\alpha})} \int_{0}^{1} \frac{\left(\xi-\frac{2}{3}\right)^{s \dot{\alpha}}}{\left[(1-\xi) \frac{\varkappa_{1}^{p}+\varkappa_{2}^{p}}{2}+\xi \varkappa_{2}^{p}\right]^{\dot{\alpha}(1-1 / p)}}(d \xi)^{\dot{\alpha}}\right)^{\frac{1}{s}} \\
& \left.\times\left(\frac{1}{\Gamma(1+\dot{\alpha})} \int_{0}^{1}\left[(1-\xi)^{\dot{\alpha}}\left|\chi^{(\dot{\alpha})}\left(\frac{\varkappa_{1}^{p}+\varkappa_{2}^{p}}{2}\right)^{1 / p}\right|^{q}+\xi^{\dot{\alpha}}\left|\chi^{(\dot{\alpha})}\left(\varkappa_{2}\right)\right|^{q}\right](d \xi)^{\dot{\alpha}}\right)^{\frac{1}{q}}\right] \\
& =\left(\frac{\varkappa_{2}^{p}-\varkappa_{1}^{p}}{4 p}\right)^{\dot{\alpha}}\left[\left(\Lambda_{7}^{(\dot{\alpha})}\left(\varkappa_{1},\left(\frac{\varkappa_{1}^{p}+\varkappa_{2}^{p}}{2}\right)^{1 / p} ; s, p\right)\right)^{\frac{1}{s}}\right. \\
& \times\left[\frac{2^{\dot{\alpha}} \Gamma(1+\dot{\alpha})}{\Gamma(1+2 \dot{\alpha})}\left(\frac{\left|\chi^{(\dot{\alpha})}\left(\varkappa_{1}\right)\right|^{q}+\left|\chi^{(\dot{\alpha})}\left(\frac{\varkappa_{1}^{p}+\varkappa_{2}^{p}}{2}\right)^{1 / p}\right|^{q}}{2^{\dot{\alpha}}}\right)\right]^{\frac{1}{q}} \\
& +\left(\Lambda_{8}^{(\dot{\alpha})}\left(\left(\frac{\varkappa_{1}^{p}+\varkappa_{2}^{p}}{2}\right)^{1 / p}, \varkappa_{2} ; s, p\right)\right)^{\frac{1}{s}} \\
& \left.\times\left[\frac{2^{\dot{\alpha}} \Gamma(1+\dot{\alpha})}{\Gamma(1+2 \dot{\alpha})}\left(\frac{\left|\chi^{(\dot{\alpha})}\left(\frac{\varkappa_{1}^{p}+\varkappa_{2}^{p}}{2}\right)^{1 / p}\right|^{q}+\left|\chi^{(\dot{\alpha})}\left(\varkappa_{2}\right)\right|^{q}}{2^{\dot{\alpha}}}\right)\right]^{\frac{1}{q}}\right] \\
& =\left(\frac{\varkappa_{2}^{p}-\varkappa_{1}^{p}}{4 p}\right)^{\dot{\alpha}}\left[\left(\Lambda_{7}^{(\dot{\alpha})}\left(\varkappa_{1},\left(\frac{\varkappa_{1}^{p}+\varkappa_{2}^{p}}{2}\right)^{1 / p} ; s, p\right)\right)^{\frac{1}{s}}\right. \\
& \times\left[\frac{2^{\dot{\alpha}} \Gamma(1+\dot{\alpha})}{\Gamma(1+2 \dot{\alpha})} \mathbb{A}^{(\dot{\alpha})}\left(\left|\chi^{(\dot{\alpha})}\left(\varkappa_{1}\right)\right|^{q},\left|\chi^{(\dot{\alpha})}\left(\frac{\varkappa_{1}^{p}+\varkappa_{2}^{p}}{2}\right)^{1 / p}\right|^{q}\right)\right]^{\frac{1}{q}} \\
& +\left(\Lambda_{8}^{(\dot{\alpha})}\left(\left(\frac{\varkappa_{1}^{p}+\varkappa_{2}^{p}}{2}\right)^{1 / p}, \varkappa_{2} ; s, p\right)\right)^{\frac{1}{s}} \\
& \left.\times\left[\frac{2^{\dot{\alpha}} \Gamma(1+\dot{\alpha})}{\Gamma(1+2 \dot{\alpha})} \mathbb{A}^{(\dot{\alpha})}\left(\left|\chi^{(\dot{\alpha})}\left(\frac{\varkappa_{1}^{p}+\varkappa_{2}^{p}}{2}\right)^{1 / p}\right|^{q},\left|\chi^{(\dot{\alpha})}\left(\varkappa_{2}\right)\right|^{q}\right)\right]^{\frac{1}{q}}\right] .
\end{aligned}
$$


Abdeljawad et al. Advances in Difference Equations

(2020) 2020:496

Page 15 of 26

Making use of Lemma 2.5 and using the change of variable technique, we get

$$
\begin{aligned}
\Lambda_{7}^{(\dot{\alpha})} & \left(\varkappa_{1},\left(\frac{\varkappa_{1}^{p}+\varkappa_{2}^{p}}{2}\right)^{1 / p} ; s, p\right) \\
:= & \frac{1}{\Gamma(1+\dot{\alpha})} \int_{0}^{1} \frac{\left|\xi-\frac{1}{3}\right|^{s \dot{\alpha}}}{\left[(1-\xi) \varkappa_{1}^{p}+\xi \frac{\varkappa_{1}^{p}+\varkappa_{2}^{p}}{2}\right]^{s \dot{\alpha}(1-1 / p)}}(d \xi)^{\dot{\alpha}} \\
= & \frac{1}{\Gamma(1+\dot{\alpha})} \int_{0}^{\frac{1}{3}} \frac{\left(\frac{1}{3}-\xi\right)^{s \dot{\alpha}}}{\left[(1-\xi) \varkappa_{1}^{p}+\xi \frac{\varkappa_{1}^{p}+\varkappa_{2}^{p}}{2}\right]^{s \dot{\alpha}(1-1 / p)}}(d \xi)^{\dot{\alpha}} \\
& +\frac{1}{\Gamma(1+\dot{\alpha})} \int_{\frac{1}{3}}^{1} \frac{\left(\xi-\frac{1}{3}\right)^{s \dot{\alpha}}}{\left[(1-\xi) \varkappa_{1}^{p}+\xi^{\frac{\varkappa_{1}^{p}+\varkappa_{2}^{p}}{2}}\right]^{s \dot{\alpha}(1-1 / p)}(d \xi)^{\dot{\alpha}}} \\
= & \left(\frac{2^{s}}{3}\right)\left(\frac{\dot{\alpha}}{\left(\varkappa_{2}^{p}-\varkappa_{1}^{p}\right)^{2 s}}\right)^{\dot{\alpha}} \\
& \times\left[\frac{1}{\Gamma(1+\dot{\alpha})} \int_{\varkappa_{1}}^{\left(\frac{5 \varkappa_{1}+\varkappa_{2}}{6}\right)^{1 / p}}\left(\frac{6^{s \dot{\alpha}}}{u^{\dot{\alpha}(p s-s-2 p+1)}}-\frac{\left(5 \varkappa_{1}^{p}+\varkappa^{p}\right)^{s \dot{\alpha}}}{u^{\dot{\alpha}(p s-s-p+1)}}\right)(d u)^{\dot{\alpha}}\right. \\
& \left.+\frac{1}{\Gamma(1+\dot{\alpha})} \int_{\left(\frac{\varkappa_{1}+\varkappa_{2}}{6}\right)^{1 / p}}^{\left(\frac{\varkappa_{1}+\varkappa_{2}}{1 / p}\right.}\left(\frac{\left(5 \varkappa_{1}^{p}+\varkappa^{p}\right)^{s \dot{\alpha}}}{u^{\dot{\alpha}(p s-s-p+1)}}-\frac{6^{s \dot{\alpha}}}{u^{\dot{\alpha}(p s-s-2 p+1)}}\right)(d u)^{\dot{\alpha}}\right] .
\end{aligned}
$$

Now, choosing $\frac{1}{u^{p s-s-2 p}}=\vartheta$ and using $\frac{(d u)^{\dot{\alpha}}}{u^{p s-s-2 p-1}}=\frac{(d \vartheta)^{\dot{\alpha}}}{p(2-s)+s}$, we obtain

$$
\begin{aligned}
& \frac{1}{\Gamma(1+\dot{\alpha})} \int_{\varkappa_{1}}^{\left(\frac{5 \varkappa_{1}+\varkappa_{2}}{6}\right)^{1 / p}} \frac{6^{s \dot{\alpha}}}{u^{\dot{\alpha}(p s-s-2 p+1)}}(d u)^{\dot{\alpha}} \\
& =\frac{6^{s \dot{\alpha}}}{(p(2-s)+s)^{\dot{\alpha}} \Gamma(1+\dot{\alpha})} \int_{\varkappa_{1}^{p(2-s)+s}}^{\left(\frac{5 \varkappa_{1}+\varkappa_{2}}{6}\right)^{(2-s)+s / p}}(d \vartheta)^{\dot{\alpha}} \\
& =\frac{6^{s \dot{\alpha}}\left(\left(\frac{5 \varkappa_{1}+\varkappa_{2}}{6}\right)^{(2-s)+s / p}-\varkappa_{1}^{p(2-s)+s}\right)^{\dot{\alpha}}}{(p(2-s)+s)^{\dot{\alpha}} \Gamma(1+\dot{\alpha})}
\end{aligned}
$$

and

$$
\begin{aligned}
& \frac{1}{\Gamma(1+\dot{\alpha})} \int_{\left(\frac{5 \varkappa_{1}+\varkappa_{2}}{6}\right)^{1 / p}}^{\left(\frac{\varkappa_{1}+\varkappa_{2}}{2}\right)^{1 / p}} \frac{6^{\dot{\alpha}}}{u^{\dot{\alpha}(p s-s-2 p+1)}}(d u)^{\dot{\alpha}} \\
& =\frac{6^{s \dot{\alpha}}}{(p(2-s)+s)^{\dot{\alpha}} \Gamma(1+\dot{\alpha})} \int_{\left(\frac{5 \varkappa_{1}+\varkappa_{2}}{6}\right)^{(2-s)+s / p}}^{\left(\frac{\varkappa_{1}+\varkappa_{2}}{2}\right)^{(2-s)+s / p}}(d \vartheta)^{\dot{\alpha}} \\
& =\frac{6^{s \dot{\alpha}}\left(\left(\frac{\varkappa_{1}+\varkappa_{2}}{2}\right)^{(2-s)+s / p}-\left(\frac{5 \varkappa_{1}+\varkappa_{2}}{6}\right)^{(2-s)+s / p}\right)^{\dot{\alpha}}}{(p(2-s)+s)^{\dot{\alpha}} \Gamma(1+\dot{\alpha})} .
\end{aligned}
$$

Again, choosing $\frac{1}{u^{p s-s-p}}=\vartheta$ and using $\frac{(d u)^{\dot{\alpha}}}{u^{p s-s-p+1}}=\frac{(d \vartheta)^{\dot{\alpha}}}{p(1-s)+s}$, we get

$$
\begin{aligned}
& \frac{\left(5 \varkappa_{1}^{p}+\varkappa^{p}\right)^{s \dot{\alpha}}}{\Gamma(1+\dot{\alpha})} \int_{\varkappa_{1}}^{\left(\frac{5 \varkappa_{1}+\varkappa_{2}}{6}\right)^{1 / p}} \frac{1}{u^{\dot{\alpha}(p s-s-p+1)}}(d u)^{\dot{\alpha}} \\
& =\frac{\left(5 \varkappa_{1}^{p}+\varkappa^{p}\right)^{s \dot{\alpha}}}{(p(1-s)+s)^{\dot{\alpha}} \Gamma(1+\dot{\alpha})} \int_{\varkappa_{1}^{p(1-s)+s}}^{\left(\frac{5 \varkappa_{1}+\varkappa_{2}}{6}\right)^{(1-s)+s / p}}(d \vartheta)^{\dot{\alpha}}
\end{aligned}
$$




$$
=\frac{\left(5 \varkappa_{1}^{p}+\varkappa^{p}\right)^{s \dot{\alpha}}\left(\left(\frac{5 \varkappa_{1}+\varkappa_{2}}{6}\right)^{(1-s)+s / p}-\varkappa_{1}^{p(1-s)+s}\right)^{\dot{\alpha}}}{(p(1-s)+s)^{\dot{\alpha}} \Gamma(1+\dot{\alpha})}
$$

and

$$
\begin{aligned}
& \frac{\left(5 \varkappa_{1}^{p}+\varkappa^{p}\right)^{s \dot{\alpha}}}{\Gamma(1+\dot{\alpha})} \int_{\left(\frac{5 \varkappa_{1}+\varkappa_{2}}{6}\right)^{1 / p}}^{\left(\frac{\varkappa_{1}+\varkappa_{2}}{2}\right)^{1 / p}} \frac{1}{u^{\dot{\alpha}(p s-s-p+1)}}(d u)^{\dot{\alpha}} \\
& =\frac{\left(5 \varkappa_{1}^{p}+\varkappa^{p}\right)^{s \dot{\alpha}}}{(p(1-s)+s)^{s \dot{\alpha}} \Gamma(1+\dot{\alpha})} \int_{\left(\frac{5 \varkappa_{1}+\varkappa_{2}}{6}\right)^{(1-s)+s / p}}^{\left(\frac{\varkappa_{1}+\varkappa_{2}}{2}\right)^{(1-s)+s / p}}(d \vartheta)^{\dot{\alpha}} \\
& =\frac{\left(5 \varkappa_{1}^{p}+\varkappa^{p}\right)^{s \dot{\alpha}}\left(\left(\frac{\varkappa_{1}+\varkappa_{2}}{2}\right)^{(1-s)+s / p}-\left(\frac{5 \varkappa_{1}+\varkappa_{2}}{6}\right)^{(1-s)+s / p}\right)^{\dot{\alpha}}}{(p(1-s)+s)^{\dot{\alpha}} \Gamma(1+\dot{\alpha})} .
\end{aligned}
$$

Substituting (3.22)-(3.25), in (3.21) we get

$$
\begin{aligned}
\Lambda_{7}^{(\dot{\alpha})}\left(\varkappa_{1},\left(\frac{\varkappa_{1}^{p}+\varkappa_{2}^{p}}{2}\right)^{1 / p} ; s, p\right) \\
:=6^{s \dot{\alpha}}\left\{\frac{\left(\left(\frac{5 \varkappa_{1}^{p}+\varkappa_{2}^{p}}{6}\right)^{(2-s)+s / p}-\varkappa_{1}^{p(2-s)+s}\right)^{\dot{\alpha}}}{(p(2-s)+s)^{\dot{\alpha}} \Gamma(1+\dot{\alpha})}\right. \\
\left.+\frac{\left(\left(\frac{\varkappa_{1}^{p}+\varkappa_{2}^{p}}{2}\right)^{(2-s)+s / p}-\left(\frac{5 \varkappa_{1}^{p}+\varkappa_{2}^{p}}{6}\right)^{(2-s)+s / p}\right)^{\dot{\alpha}}}{(p(2-s)+s)^{\dot{\alpha}} \Gamma(1+\dot{\alpha})}\right\} \\
-\left(5 \varkappa_{1}^{p}+\varkappa^{p}\right)^{s \dot{\alpha}}\left\{\frac{\left(\left(\frac{5 \varkappa_{1}^{p}+\varkappa_{2}^{p}}{6}\right)^{(1-s)+s / p}-\varkappa_{1}^{p(1-s)+s}\right)^{\dot{\alpha}}}{(p(1-s)+s)^{\dot{\alpha}} \Gamma(1+\dot{\alpha})}\right. \\
\left.+\frac{\left(\left(\frac{\varkappa_{1}^{p}+\varkappa_{2}^{p}}{2}\right)^{(1-s)+s / p}-\left(\frac{5 \varkappa_{1}^{p}+\varkappa_{2}^{p}}{6}\right)^{(1-s)+s / p}\right)^{\dot{\alpha}}}{(p(1-s)+s)^{\dot{\alpha}} \Gamma(1+\dot{\alpha})}\right\} .
\end{aligned}
$$

Analogously,

$$
\begin{aligned}
\Lambda_{8}^{(\dot{\alpha})} & \left(\left(\frac{\varkappa_{1}^{p}+\varkappa_{2}^{p}}{2}\right)^{1 / p}, \varkappa_{2} ; s, p\right) \\
:= & \left(\varkappa_{1}^{p}+5 \varkappa^{p}\right)^{s \dot{\alpha}}\left\{\frac{\left(\left(\frac{\varkappa_{1}^{p}+5 \varkappa_{2}^{p}}{6}\right)^{(1-s)+s / p}-\left(\frac{\varkappa_{1}^{p}+\varkappa_{2}^{p}}{2}\right)^{(1-s)+s / p}\right)^{\dot{\alpha}}}{(p(1-s)+s)^{\dot{\alpha}} \Gamma(1+\dot{\alpha})}\right. \\
& \left.+\frac{\left.\varkappa_{2}^{p(1-s)+s}-\left(\frac{\varkappa_{1}^{p}+5 \varkappa_{2}^{p}}{6}\right)^{(1-s)+s / p}\right)^{\dot{\alpha}}}{(p(1-s)+s)^{\dot{\alpha}} \Gamma(1+\dot{\alpha})}\right\} \\
& -6^{s \dot{\alpha}}\left\{\frac{\left.\left(\left(\frac{\varkappa_{1}^{p}+5 \varkappa_{2}^{p}}{6}\right)^{(2-s)+s / p}-\left(\frac{\varkappa_{1}^{p}+\varkappa_{2}^{p}}{2}\right)^{(2-s)+s / p}\right)^{\dot{\alpha}}\right)^{\dot{\alpha}}}{(p(2-s)+s)^{\dot{\alpha}} \Gamma(1+\dot{\alpha})}\right. \\
& \left.-\frac{\left.\left(\varkappa_{2}^{p(2-s)+s}-\left(\frac{\varkappa_{1}^{p}+5 \varkappa_{2}^{p}}{6}\right)^{(2-s)+s / p}\right)^{\dot{\alpha}}\right]}{(p(2-s)+s)^{\dot{\alpha}} \Gamma(1+\dot{\alpha})}\right\} .
\end{aligned}
$$

Putting the values of $\Lambda_{7}^{(\dot{\alpha})}\left(\varkappa_{1},\left(\frac{\varkappa_{1}^{p}+\varkappa_{2}^{p}}{2}\right)^{1 / p} ; s, p\right)$ and $\Lambda_{8}^{(\dot{\alpha})}\left(\left(\frac{\varkappa_{1}^{p}+\varkappa_{2}^{p}}{2}\right)^{1 / p}, \varkappa_{2} ; s, p\right)$ in (3.20), we get the desired inequality (3.17). This completes the proof.

Remark 3.8 Theorem 3.7 leads to the following conclusions:

(1) If $\dot{\alpha}=1$, then we get Theorem 2.2 of [29]. 
Abdeljawad et al. Advances in Difference Equations

(2020) $2020: 496$

Page 17 of 26

(2) If $\dot{\alpha}=p=1$, then we get Theorem 8 of [28] when we choose $s=1$, which coincides with the first part of Corollary 2.2 of [29].

(3) If $\dot{\alpha}=1$ and $p=-1$, then we get the second part of Corollary 2.2 of [29].

Theorem 3.9 Let $p \in \mathbb{R} \backslash\{0\}, s, q>1$ with $s^{-1}+q^{-1}=1$ and $\chi: \Omega^{\circ} \subset \mathbb{R} \rightarrow \mathbb{R}^{\dot{\alpha}}$ be a function such that $\chi \in \mathcal{D}_{\dot{\alpha}}\left(\Omega^{\circ}\right)\left(\Omega^{\circ}\right.$ is the interior of $\left.\Omega\right), \chi^{(\dot{\alpha})} \in \mathcal{C}_{\dot{\alpha}}\left[\varkappa_{1}, \varkappa_{2}\right]$ and $\left|\chi^{(\dot{\alpha})}\right|^{q}$ is a generalized p-convex function on $\Omega$. Then

$$
\begin{aligned}
& \mid\left(\frac{1}{6}\right)^{\dot{\alpha}}\left[\chi\left(\varkappa_{1}\right)+(4)^{\dot{\alpha}} \chi\left(\left[\frac{\varkappa_{1}^{p}+\varkappa_{2}^{p}}{2}\right]^{1 / p}\right)+\chi\left(\varkappa_{2}\right)\right] \\
& \quad-\left(\frac{2 p}{\varkappa_{2}^{p}-\varkappa_{1}^{p}}\right)^{\dot{\alpha}} \varkappa_{1} \mathcal{I}_{\varkappa_{2}}^{(\dot{\alpha})} \frac{\chi(u)}{u^{\dot{\alpha}(1-p)}} \mid \\
& \leq\left(\frac{\varkappa_{2}^{p}-\varkappa_{1}^{p}}{4 p}\right)^{\dot{\alpha}}\left[[ \frac { \Gamma ( 1 + \dot { \alpha } s ) } { \Gamma ( 1 + ( s + 1 ) \dot { \alpha } ) } ] ^ { \frac { 1 } { s } } \left[\Theta_{1}^{(\dot{\alpha})}\left(\varkappa_{1},\left(\frac{\varkappa_{1}^{p}+\varkappa_{2}^{p}}{2}\right)^{1 / p} ; q, p\right)\left|\chi^{(\dot{\alpha})}\left(\varkappa_{1}\right)\right|^{q}\right.\right. \\
& \left.\left.\quad+\Theta_{2}^{(\dot{\alpha})}\left(\varkappa_{1},\left(\frac{\varkappa_{1}^{p}+\varkappa_{2}^{p}}{2}\right)^{1 / p} ; q, p\right)\left|\chi^{(\dot{\alpha})}\left(\frac{\varkappa_{1}^{p}+\varkappa_{2}^{p}}{2}\right)^{1 / p}\right|^{q}\right]^{\frac{1}{q}}\right]^{\frac{1}{s}}\left[\Theta_{3}^{(\dot{\alpha})}\left(\left(\frac{\varkappa_{1}^{p}+\varkappa_{2}^{p}}{2}\right)^{1 / p}, \varkappa_{2} ; q, p\right)\left|\chi^{(\dot{\alpha})}\left(\frac{\varkappa_{1}^{p}+\varkappa_{2}^{p}}{2}\right)^{1 / p}\right|^{q}\right. \\
& \left.\quad+\left[\frac{\Gamma(1+\dot{\alpha} s)}{\Gamma(1+(s+1) \dot{\alpha})}\right]^{\frac{1}{q}}\left|\chi^{(\dot{\alpha})}\left(\varkappa_{2}\right)\right|^{q}\right] \\
& \left.\quad+\Theta_{4}^{(\dot{\alpha})}\left(\left(\frac{\varkappa_{1}^{p}+\varkappa_{2}^{p}}{2}\right)^{1 / p}, \varkappa_{2} ; q, p\right)\right]^{(3)}
\end{aligned}
$$

where

$$
\begin{aligned}
\Theta_{1}^{(\dot{\alpha})} & \left(\varkappa_{1},\left(\frac{\varkappa_{1}^{p}+\varkappa_{2}^{p}}{2}\right)^{1 / p} ; q, p\right) \\
:= & \left(\frac{2 p}{\left(\varkappa_{2}^{p}-\varkappa_{1}^{p}\right)^{2}}\right)^{\dot{\alpha}}\left[\frac{\left(\varkappa_{1}^{p}+\varkappa_{2}^{p}\right)^{\dot{\alpha}}}{(p(1-q)+q)^{\dot{\alpha}} \Gamma(1+\dot{\alpha})}\right. \\
& \times\left(\left(\frac{\varkappa_{1}^{p}+\varkappa_{2}^{p}}{2}\right)^{(1-q)+q / p}-\varkappa_{1}^{p(1-q)+q}\right)^{\dot{\alpha}} \\
& \left.-\frac{22^{\dot{\alpha}}}{(p(2-q)+q)^{\dot{\alpha}} \Gamma(1+\dot{\alpha})}\left(\left(\frac{\varkappa_{1}^{p}+\varkappa_{2}^{p}}{2}\right)^{(2-q)+q / p}-\varkappa_{1}^{p(2-q)+q}\right)^{\dot{\alpha}}\right], \\
\Theta_{2}^{(\dot{\alpha})} & \left(\varkappa_{1},\left(\frac{\varkappa_{1}^{p}+\varkappa_{2}^{p}}{2}\right)^{1 / p} ; q, p\right) \\
:= & \left(\frac{4 p}{\left(\varkappa_{2}^{p}-\varkappa_{1}^{p}\right)^{2}}\right)^{\dot{\alpha}}\left[\frac{1}{(p(1-q)+q)^{\dot{\alpha}} \Gamma(1+\dot{\alpha})}\right. \\
& \times\left(\left(\frac{\varkappa_{1}^{p}+\varkappa_{2}^{p}}{2}\right)^{(1-q)+q / p}-\varkappa_{1}^{p(1-q)+q}\right)^{\dot{\alpha}} \\
& \left.-\frac{\left(\varkappa_{1}^{p}\right)^{\dot{\alpha}}}{(p(2-q)+q)^{\dot{\alpha}} \Gamma(1+\dot{\alpha})}\left(\left(\frac{\varkappa_{1}^{p}+\varkappa_{2}^{p}}{2}\right)^{(2-q)+q / p}-\varkappa_{1}^{p(2-q)+q}\right)^{\dot{\alpha}}\right], \\
\Theta_{3}^{(\dot{\alpha})}( & \left.\left(\frac{\varkappa_{1}^{p}+\varkappa_{2}^{p}}{2}\right)^{1 / p} ; \varkappa_{2}, q, p\right) \\
:= & \left.\frac{4 p}{\left(\varkappa_{2}^{p}-\varkappa_{1}^{p}\right)^{2}}\right)^{\dot{\alpha}}\left[\frac{\left(\varkappa_{2}^{p}\right)^{\dot{\alpha}}}{(p(1-q)+q)^{\dot{\alpha}} \Gamma(1+\dot{\alpha})}\right.
\end{aligned}
$$


Abdeljawad et al. Advances in Difference Equations

(2020) 2020:496

Page 18 of 26

$$
\begin{aligned}
& \times\left(\varkappa_{2}^{p(1-q)+q}-\left(\frac{\varkappa_{1}^{p}+\varkappa_{2}^{p}}{2}\right)^{(1-q)+q / p}\right)^{\dot{\alpha}} \\
& \left.-\frac{1}{(p(2-q)+q)^{\dot{\alpha}} \Gamma(1+\dot{\alpha})}\left(\varkappa_{2}^{p(1-q)+q}-\left(\frac{\varkappa_{1}^{p}+\varkappa_{2}^{p}}{2}\right)^{(2-q)+q / p}\right)^{\dot{\alpha}}\right],
\end{aligned}
$$

and

$$
\begin{aligned}
\Theta_{4}^{(\dot{\alpha})} & \left(\varkappa_{1},\left(\frac{\varkappa_{1}^{p}+\varkappa_{2}^{p}}{2}\right)^{1 / p}, \varkappa_{2} ; q, p\right) \\
:= & \left(\frac{2 p}{\left(\varkappa_{2}^{p}-\varkappa_{1}^{p}\right)^{2}}\right)^{\dot{\alpha}}\left[\frac{(2)^{\dot{\alpha}}}{(p(1-q)+q)^{\dot{\alpha}} \Gamma(1+\dot{\alpha})}\right. \\
& \times\left(\varkappa_{2}^{p(1-q)+q}-\left(\frac{\varkappa_{1}^{p}+\varkappa_{2}^{p}}{2}\right)^{(1-q)+q / p}\right)^{\dot{\alpha}} \\
& \left.-\frac{\left(\varkappa_{1}^{p}+\varkappa_{2}^{p}\right)^{\dot{\alpha}}}{(p(2-q)+q)^{\dot{\alpha}} \Gamma(1+\dot{\alpha})}\left(\varkappa_{2}^{p(1-q)+q}-\left(\frac{\varkappa_{1}^{p}+\varkappa_{2}^{p}}{2}\right)^{(2-q)+q / p}\right)^{\dot{\alpha}}\right] .
\end{aligned}
$$

Proof It follows from Lemma 3.4 and the generalized Hölder inequality that

$$
\begin{aligned}
& \mid\left(\frac{1}{6}\right)^{\dot{\alpha}}\left[\chi\left(\varkappa_{1}\right)+(4)^{\dot{\alpha}} \chi\left(\left[\frac{\varkappa_{1}^{p}+\varkappa_{2}^{p}}{2}\right]^{1 / p}\right)+\chi\left(\varkappa_{2}\right)\right] \\
& -\left(\frac{2 p}{\varkappa_{2}^{p}-\varkappa_{1}^{p}}\right)^{\dot{\alpha}} \varkappa_{1} \mathcal{I}_{\varkappa_{2}}^{(\dot{\alpha})} \frac{\chi(u)}{u^{\dot{\alpha}(1-p)}} \mid \\
& \leq\left(\frac{\varkappa_{2}^{p}-\varkappa_{1}^{p}}{4 p}\right)^{\dot{\alpha}}\left[\left(\frac{1}{\Gamma(1+\dot{\alpha})} \int_{0}^{1}\left|\xi-\frac{1}{3}\right|^{s \dot{\alpha}}(d \xi)^{\dot{\alpha}}\right)^{\frac{1}{s}}\right. \\
& \times\left(\frac{1}{\Gamma(1+\dot{\alpha})} \int_{0}^{1} \frac{\left|\chi^{(\dot{\alpha})}\left(\left[(1-\xi) \varkappa_{1}^{p}+\xi \frac{\varkappa_{1}^{p}+\varkappa_{2}^{p}}{2}\right]^{1 / p}\right)\right|^{q}}{\left[(1-\xi) \varkappa_{1}^{p}+\xi \frac{\varkappa_{1}^{p}+\varkappa_{2}^{p}}{2}\right]^{q \dot{\alpha}(1-1 / p)}}(d \xi)^{\dot{\alpha}}\right)^{\frac{1}{q}} \\
& +\left(\frac{1}{\Gamma(1+\dot{\alpha})} \int_{0}^{1}\left(\xi-\frac{2}{3}\right)^{s \dot{\alpha}}(d \xi)^{\dot{\alpha}}\right)^{\frac{1}{s}} \\
& \left.\times\left(\frac{1}{\Gamma(1+\dot{\alpha})} \int_{0}^{1} \frac{\left|\chi^{(\dot{\alpha})}\left(\left[(1-\xi) \frac{\varkappa_{1}^{p}+\varkappa_{2}^{p}}{2}+\xi \varkappa_{2}^{p}\right]^{1 / p}\right)\right|^{q}}{\left[(1-\xi) \frac{\varkappa_{1}^{p}+\varkappa_{2}^{p}}{2}+\xi \varkappa_{2}^{p}\right]^{q \dot{\alpha}(1-1 / p)}}(d \xi)^{\dot{\alpha}}\right)^{\frac{1}{q}}\right] \text {. }
\end{aligned}
$$

By the generalized $p$-convexity of $\left|\chi^{(\dot{\alpha})}\right|^{q}$ on $\Omega$, we have

$$
\begin{aligned}
& \mid\left(\frac{1}{6}\right)^{\dot{\alpha}}\left[\chi\left(\varkappa_{1}\right)+(4)^{\dot{\alpha}} \chi\left(\left[\frac{\varkappa_{1}^{p}+\varkappa_{2}^{p}}{2}\right]^{1 / p}\right)+\chi\left(\varkappa_{2}\right)\right] \\
& -\left(\frac{2 p}{\varkappa_{2}^{p}-\varkappa_{1}^{p}}\right)^{\dot{\alpha}} \varkappa_{1} \mathcal{I}_{\varkappa_{2}}^{(\dot{\alpha})} \frac{\chi(u)}{u^{\dot{\alpha}(1-p)}} \mid \\
& \leq\left(\frac{\varkappa_{2}^{p}-\varkappa_{1}^{p}}{4 p}\right)^{\dot{\alpha}}\left[\left(\frac{1}{\Gamma(1+\dot{\alpha})} \int_{0}^{1}\left|\xi-\frac{1}{3}\right|^{s \dot{\alpha}}(d \xi)^{\dot{\alpha}}\right)^{\frac{1}{s}}\right. \\
& \times\left(\frac{1}{\Gamma(1+\dot{\alpha})} \int_{0}^{1} \frac{\left[(1-\xi)^{\dot{\alpha}}\left|\chi^{(\dot{\alpha})}\left(\varkappa_{1}\right)\right|^{q}+\xi^{\dot{\alpha}}\left|\chi^{(\dot{\alpha})}\left(\frac{\varkappa_{1}^{p}+\varkappa_{2}^{p}}{2}\right)^{1 / p}\right|^{q}\right]}{\left[(1-\xi) \varkappa_{1}^{p}+\xi \frac{\varkappa_{1}^{p}+\varkappa_{2}^{p}}{2}\right]^{q \dot{\alpha}(1-1 / p)}}(d \xi)^{\dot{\alpha}}\right)^{\frac{1}{q}}
\end{aligned}
$$




$$
\begin{aligned}
& +\left(\frac{1}{\Gamma(1+\dot{\alpha})} \int_{0}^{1}\left(\xi-\frac{2}{3}\right)^{s \dot{\alpha}}(d \xi)^{\dot{\alpha}}\right)^{\frac{1}{s}} \\
& \left.\times\left(\frac{1}{\Gamma(1+\dot{\alpha})} \int_{0}^{1} \frac{\left[(1-\xi)^{\dot{\alpha}}\left|\chi^{(\dot{\alpha})}\left(\frac{\varkappa_{1}^{p}+\varkappa_{2}^{p}}{2}\right)^{1 / p}\right|^{q}+\xi^{\dot{\alpha}}\left|\chi^{(\dot{\alpha})}\left(\varkappa_{2}\right)\right|^{q}\right]}{\left[(1-\xi) \frac{\varkappa_{1}^{p}+\varkappa_{2}^{p}}{2}+\xi \varkappa_{2}^{p}\right]^{\dot{\alpha}(1-1 / p)}}(d \xi)^{\dot{\alpha}}\right)^{\frac{1}{q}}\right] \\
& =\left(\frac{\varkappa_{2}^{p}-\varkappa_{1}^{p}}{4 p}\right)^{\dot{\alpha}}\left[( \Theta _ { 1 } ^ { ( \dot { \alpha } ) } ( s ) ^ { \frac { 1 } { s } } ) \left[\Theta_{2}^{(\dot{\alpha})}\left(\varkappa_{1},\left(\frac{\varkappa_{1}^{p}+\varkappa_{2}^{p}}{2}\right)^{1 / p} ; q, p\right)\left|\chi^{(\dot{\alpha})}\left(\varkappa_{1}\right)\right|^{q}\right.\right. \\
& \left.+\Theta_{3}^{(\dot{\alpha})}\left(\varkappa_{1},\left(\frac{\varkappa_{1}^{p}+\varkappa_{2}^{p}}{2}\right)^{1 / p} ; q, p\right)\left|\chi^{(\dot{\alpha})}\left(\frac{\varkappa_{1}^{p}+\varkappa_{2}^{p}}{2}\right)^{1 / p}\right|^{q}\right]^{\frac{1}{q}} \\
& +\left(\Theta_{1}^{(\dot{\alpha})}(s)^{\frac{1}{s}}\right)\left[\Theta_{4}^{(\dot{\alpha})}\left(\left(\frac{\varkappa_{1}^{p}+\varkappa_{2}^{p}}{2}\right)^{1 / p}, \varkappa_{2} ; q, p\right)\left|\chi^{(\dot{\alpha})}\left(\frac{\varkappa_{1}^{p}+\varkappa_{2}^{p}}{2}\right)^{1 / p}\right|^{q}\right. \\
& \left.\left.+\Theta_{5}^{(\dot{\alpha})}\left(\left(\frac{\varkappa_{1}^{p}+\varkappa_{2}^{p}}{2}\right)^{1 / p}, \varkappa_{2} ; q, p\right)\right]^{\frac{1}{q}}\left|\chi^{(\dot{\alpha})}\left(\varkappa_{2}\right)\right|^{q}\right] \text {. }
\end{aligned}
$$

From Lemma 2.5 we clearly see that

$$
\frac{1}{\Gamma(1+\dot{\alpha})} \int_{0}^{1}\left|\xi-\frac{1}{3}\right|^{s \dot{\alpha}}(d \xi)^{\dot{\alpha}}=\frac{1}{\Gamma(1+\dot{\alpha})} \int_{0}^{1}\left|\xi-\frac{2}{3}\right|^{s \dot{\alpha}}(d \xi)^{\dot{\alpha}}=\frac{\Gamma(1+s \dot{\alpha})}{\Gamma(1+(s+1) \dot{\alpha})}
$$

Again, using Lemma 2.5 and the change of the variable technique, we have

$$
\begin{aligned}
& \Theta_{1}^{(\dot{\alpha})}\left(\varkappa_{1},\left(\frac{\varkappa_{1}^{p}+\varkappa_{2}^{p}}{2}\right)^{1 / p} ; q, p\right) \\
& \quad:=\frac{1}{\Gamma(1+\dot{\alpha})} \int_{0}^{1} \frac{(1-\xi)^{\dot{\alpha}}(d \xi)^{\dot{\alpha}}}{\left[(1-\xi) \varkappa_{1}^{p}+\xi \frac{\varkappa_{1}^{p}+\varkappa_{2}^{p}}{2}\right]^{q \dot{\alpha}(1-1 / p)}} \\
& \quad=\left(\frac{2 p}{\left(\varkappa_{2}^{p}-\varkappa_{1}^{p}\right)^{2}}\right)^{\dot{\alpha}} \frac{1}{\Gamma(1+\dot{\alpha})} \int_{\varkappa_{1}}^{\left(\frac{\varkappa_{1}+\varkappa_{2}}{2}\right)^{\frac{1}{p}}}\left(\frac{\left(\varkappa_{1}^{p}+\varkappa_{2}^{p}\right)^{\dot{\alpha}}}{u^{\dot{\alpha}(p q-q-p+1)}}-\frac{2^{\dot{\alpha}}}{u^{\dot{\alpha}(p q-q-2 p+1)}}\right)(d u)^{\dot{\alpha}} .
\end{aligned}
$$

Now, choosing $\frac{1}{u^{p q-q-p}}=\vartheta$ and using $\frac{(d u)^{\dot{\alpha}}}{u^{p q-q-p+1}}=\frac{\left(d \vartheta \vartheta^{\dot{\alpha}}\right.}{(p(1-q)+q)^{\dot{\alpha}}}$, we obtain

$$
\begin{aligned}
& \frac{\left(\varkappa_{1}^{p}+\varkappa_{\varkappa^{p}}^{p}\right)^{\dot{\alpha}}}{(p(1-q)+q)^{\dot{\alpha}} \Gamma(1+\dot{\alpha})} \int_{\varkappa_{1}}^{\left(\frac{\varkappa_{1}^{p}+\varkappa_{2}^{p}}{2}\right)^{\frac{1}{p}}} \frac{1}{u^{\dot{\alpha}(p q-q-p+1)}}(d u)^{\dot{\alpha}} \\
& =\frac{\left(\varkappa_{1}^{p}+\varkappa_{2}^{p}\right)^{\dot{\alpha}}}{(p(1-q)+q)^{\dot{\alpha}} \Gamma(1+\dot{\alpha})} \int_{\varkappa_{1}^{p(1-q)+q}}^{\left(\frac{\varkappa_{1}^{p}+\varkappa_{2}^{p}}{2}\right)^{(1-q)+q / p}}(d \vartheta)^{\dot{\alpha}} \\
& =\frac{\left(\varkappa_{1}^{p}+\varkappa_{2}^{p}\right)^{\dot{\alpha}}}{(p(1-q)+q)^{\dot{\alpha}} \Gamma(1+\dot{\alpha})}\left(\left(\frac{\varkappa_{1}^{p}+\varkappa_{2}^{p}}{2}\right)^{(1-q)+q / p}-\varkappa_{1}^{p(1-q)+q}\right)^{\dot{\alpha}} .
\end{aligned}
$$

Again, choosing $\frac{1}{u^{p q-q-2 p}}=\vartheta$ and from $\frac{(d u)^{\dot{\alpha}}}{u^{p q-q-2 p+1}}=\frac{(d \vartheta)^{\dot{\alpha}}}{(p(2-q)+q)^{\dot{\alpha}}}$, we obtain

$$
\frac{2^{\dot{\alpha}}}{(p(2-q)+q)^{\dot{\alpha}} \Gamma(1+\dot{\alpha})} \int_{\varkappa_{1}}^{\left(\frac{\varkappa_{1}^{p}+\varkappa_{2}^{p}}{2}\right)^{\frac{1}{p}}} \frac{1}{u^{\dot{\alpha}(p q-q-p+1)}}(d u)^{\dot{\alpha}}
$$




$$
\begin{aligned}
& =\frac{2^{\dot{\alpha}}}{(p(2-q)+q)^{\dot{\alpha}} \Gamma(1+\dot{\alpha})} \int_{\varkappa_{1}^{p(2-q)+q}}^{\left(\frac{\varkappa_{1}^{p}+\varkappa_{2}^{p}}{2}\right)^{(2-q)+q / p}(d \vartheta)^{\dot{\alpha}}} \\
& =\frac{2^{\dot{\alpha}}}{(p(2-q)+q)^{\dot{\alpha}} \Gamma(1+\dot{\alpha})}\left(\left(\frac{\varkappa_{1}^{p}+\varkappa_{2}^{p}}{2}\right)^{(2-q)+q / p}-\varkappa_{1}^{p(2-q)+q}\right)^{\dot{\alpha}} .
\end{aligned}
$$

After substituting (3.34) and (3.35) in (3.33), we get

$$
\begin{aligned}
\Theta_{1}^{(\dot{\alpha})} & \left(\varkappa_{1},\left(\frac{\varkappa_{1}^{p}+\varkappa_{2}^{p}}{2}\right)^{1 / p} ; q, p\right) \\
:= & \left(\frac{2 p}{\left(\varkappa_{2}^{p}-\varkappa_{1}^{p}\right)^{2}}\right)^{\dot{\alpha}}\left[\frac{\left(\varkappa_{1}^{p}+\varkappa_{2}^{p}\right)^{\dot{\alpha}}}{(p(1-q)+q)^{\dot{\alpha}} \Gamma(1+\dot{\alpha})}\right. \\
& \times\left(\left(\frac{\varkappa_{1}^{p}+\varkappa_{2}^{p}}{2}\right)^{(1-q)+q / p}-\varkappa_{1}^{p(1-q)+q}\right)^{\dot{\alpha}} \\
& \left.-\frac{2^{\dot{\alpha}}}{(p(2-q)+q)^{\dot{\alpha}} \Gamma(1+\dot{\alpha})}\left(\left(\frac{\varkappa_{1}^{p}+\varkappa_{2}^{p}}{2}\right)^{(2-q)+q / p}-\varkappa_{1}^{p(2-q)+q}\right)^{\dot{\alpha}}\right],
\end{aligned}
$$

where we have used the identities

$$
\begin{aligned}
& \Theta_{2}^{(\dot{\alpha})}\left(\varkappa_{1},\left(\frac{\varkappa_{1}^{p}+\varkappa_{2}^{p}}{2}\right)^{1 / p} ; q, p\right):=\frac{1}{\Gamma(1+\dot{\alpha})} \int_{0}^{1} \frac{\xi^{\dot{\alpha}}(d \xi)^{\dot{\alpha}}}{\left[(1-\xi) \varkappa_{1}^{p}+\xi \frac{\varkappa_{1}^{p}+\varkappa_{2}^{p}}{2}\right]^{q \dot{\alpha}(1-1 / p)}}, \\
& \Theta_{3}^{(\dot{\alpha})}\left(\left(\frac{\varkappa_{1}^{p}+\varkappa_{2}^{p}}{2}\right)^{1 / p} ; \varkappa_{2}, q, p\right):=\frac{1}{\Gamma(1+\dot{\alpha})} \int_{0}^{1} \frac{(1-\xi)^{\dot{\alpha}}(d \xi)^{\dot{\alpha}}}{\left[(1-\xi) \frac{\varkappa_{1}^{p}+\varkappa_{2}^{p}}{2}+\xi \varkappa_{2}\right]^{q \dot{\alpha}(1-1 / p)}}, \\
& \Theta_{4}^{(\dot{\alpha})}\left(\varkappa_{1},\left(\frac{\varkappa_{1}^{p}+\varkappa_{2}^{p}}{2}\right)^{1 / p}, \varkappa_{2} ; q, p\right):=\frac{1}{\Gamma(1+\dot{\alpha})} \int_{0}^{1} \frac{\xi^{\dot{\alpha}}(d \xi)^{\dot{\alpha}}}{\left[(1-\xi) \frac{\varkappa_{1}^{p}+\varkappa_{2}^{p}}{2}+\xi \varkappa_{2}\right]^{q \dot{\alpha}(1-1 / p)}} .
\end{aligned}
$$

Substituting (3.32)-(3.37) in (3.31), we get the desired inequality (3.26). This completes the proof.

Remark 3.10 Theorem 3.9 leads to the following conclusions:

(1) Let $\dot{\alpha}=1$. Then we get Theorem 2.3 of [29].

(2) Let $\dot{\alpha}=p=1$. Then we get the first part of Corollary 2.3 of [29].

(3) Let $\dot{\alpha}=1$ and $p=-1$. Then we get the second part of Corollary 2.3 of [29].

\section{Applications}

\subsection{Probability density functions}

Consider a random variable $\mathcal{Z}$ whose generalized probability density function is $\mathfrak{p}$ : $\left[\varkappa_{1}, \varkappa_{2}\right] \rightarrow\left[0^{\dot{\alpha}}, 1^{\dot{\alpha}}\right]$, which is generalized convex with the cumulative distribution function $\mathcal{F}_{\dot{\alpha}}$ defined by

$$
P_{\dot{\alpha}}(\mathcal{Z} \leq x)=\mathcal{F}_{\dot{\alpha}}(u):=\frac{1}{\Gamma(1+\dot{\alpha})} \int_{\varkappa_{1}}^{u} \mathfrak{p}(\xi)(d \xi)^{\dot{\alpha}} .
$$

Moreover, the generalized expectation can be expressed as

$$
E_{\dot{\alpha}}=\frac{1}{\Gamma(1+\dot{\alpha})} \int_{\varkappa_{1}}^{\varkappa_{2}} \xi^{\dot{\alpha}} \mathfrak{p}(\xi)(d \xi)^{\dot{\alpha}} .
$$


We clearly see that

$$
E_{\dot{\alpha}}(u)=\varkappa_{2}^{\dot{\alpha}}-\frac{1}{\Gamma(1+\dot{\alpha})} \int_{\varkappa_{1}}^{\varkappa_{2}} \mathcal{F}_{\dot{\alpha}}(\xi)(d \xi)^{\dot{\alpha}}
$$

The following two propositions can be obtained from our results obtained in Sect. 3 immediately.

Proposition 4.1 Let $p \in \mathbb{R} \backslash\{0\}$. Then Theorem 3.5 leads to

$$
\begin{aligned}
& \mid\left(\frac{1}{6}\right)^{\dot{\alpha}}\left[P_{\dot{\alpha}}\left(\mathcal{Y} \leq \varkappa_{1}\right)+(4)^{\dot{\alpha}} P_{\dot{\alpha}}\left(\mathcal{Y} \leq\left(\left[\frac{\varkappa_{1}^{p}+\varkappa_{2}^{p}}{2}\right]^{1 / p}\right)+P_{\dot{\alpha}}\left(\mathcal{Y} \leq \varkappa_{2}\right)\right]\right. \\
& \quad-\left(\frac{2}{\varkappa_{2}^{p}-\varkappa_{1}^{p}}\right)^{\dot{\alpha}}\left(\varkappa_{2}-E_{\dot{\alpha}}(\mathcal{Y})\right) \mid \\
& \leq\left(\frac{\varkappa_{2}^{p}-\varkappa_{1}^{p}}{4 p}\right)^{\dot{\alpha}}\left[\left(\Lambda_{1}^{(\dot{\alpha})}\left(\left(\frac{\varkappa_{1}^{p}+\varkappa_{2}^{p}}{2}\right)^{1 / p}, \varkappa_{1} ; p\right)\right)^{1-\frac{1}{q}}\right. \\
& \quad \times\left[\Lambda_{2}^{(\dot{\alpha})}\left(\left(\frac{\varkappa_{1}^{p}+\varkappa_{2}^{p}}{2}\right)^{1 / p}, \varkappa_{1} ; p\right)\left|\mathfrak{p}\left(\varkappa_{1}\right)\right|^{q}\right. \\
& \left.+\Lambda_{3}^{(\dot{\alpha})}\left(\left(\frac{\varkappa_{1}^{p}+\varkappa_{2}^{p}}{2}\right)^{1 / p}, \varkappa_{1} ; p\right)\left|\mathfrak{p}\left(\frac{\varkappa_{1}^{p}+\varkappa_{2}^{p}}{2}\right)^{1 / p}\right|^{q}\right]^{\frac{1}{q}} \\
& \quad+\left(\Lambda_{4}^{(\dot{\alpha})}\left(\left(\frac{\varkappa_{1}^{p}+\varkappa_{2}^{p}}{2}\right)^{1 / p}, \varkappa_{2} ; p\right)\right)^{1-\frac{1}{q}} \\
& \quad \times\left[\Lambda_{5}^{(\dot{\alpha})}\left(\left(\frac{\varkappa_{1}^{p}+\varkappa_{2}^{p}}{2}\right)^{1 / p}, \varkappa_{2} ; p\right)\left|\mathfrak{p}\left(\frac{\varkappa_{1}^{p}+\varkappa_{2}^{p}}{2}\right)^{1 / p}\right|^{q}\right. \\
& \left.\left.\quad+\Lambda_{6}^{(\dot{\alpha})}\left(\left(\frac{\varkappa_{1}^{p}+\varkappa_{2}^{p}}{2}\right)^{1 / p}, \varkappa_{2} ; p\right)\left|\mathfrak{p}\left(\varkappa_{2}\right)\right|^{q}\right]^{\frac{1}{q}}\right],
\end{aligned}
$$

where $\quad \Lambda_{1}^{(\dot{\alpha})}\left(\varkappa_{1},\left(\frac{\varkappa_{1}^{p}+\varkappa_{2}^{p}}{2}\right)^{1 / p} ; p\right), \quad \Lambda_{2}^{(\dot{\alpha})}\left(\varkappa_{1},\left(\frac{\varkappa_{1}^{p}+\varkappa_{2}^{p}}{2}\right)^{1 / p}, \varkappa_{1} ; p\right), \quad \Lambda_{3}^{(\dot{\alpha})}\left(\varkappa_{1},\left(\frac{\varkappa_{1}^{p}+\varkappa_{2}^{p}}{2}\right)^{1 / p} ; p\right)$, $\Lambda_{4}^{(\dot{\alpha})}\left(\left(\frac{\varkappa_{1}^{p}+\varkappa_{2}^{p}}{2}\right)^{1 / p}, \varkappa_{2} ; p\right), \Lambda_{5}^{(\dot{\alpha})}\left(\left(\frac{\varkappa_{1}^{p}+\varkappa_{2}^{p}}{2}\right)^{1 / p}, \varkappa_{2} ; p\right)$, and $\Lambda_{6}^{(\dot{\alpha})}\left(\left(\frac{\varkappa_{1}^{p}+\varkappa_{2}^{p}}{2}\right)^{1 / p}, \varkappa_{2} ; p\right)$ are given in $(3.6)-$ (3.10), respectively.

Proposition 4.2 Let $p \in \mathbb{R} \backslash\{0\}$. Then Theorem 3.7 leads to the conclusion that

$$
\begin{aligned}
& \mid\left(\frac{1}{6}\right)^{\dot{\alpha}} {\left[P_{\dot{\alpha}}\left(\mathcal{Y} \leq \varkappa_{1}\right)+(4)^{\dot{\alpha}} P_{\dot{\alpha}}\left(\mathcal{Y} \leq\left(\left[\frac{\varkappa_{1}^{p}+\varkappa_{2}^{p}}{2}\right]^{1 / p}\right)+P_{\dot{\alpha}}\left(\mathcal{Y} \leq \varkappa_{2}\right)\right]\right.} \\
&-\left(\frac{2}{\varkappa_{2}^{p}-\varkappa_{1}^{p}}\right)^{\dot{\alpha}}\left(\varkappa_{2}-E_{\dot{\alpha}}(\mathcal{Y})\right) \mid \\
& \leq\left(\frac{\varkappa_{2}^{p}-\varkappa_{1}^{p}}{4 p}\right)^{\dot{\alpha}}\left[\left(\Lambda_{7}^{(\dot{\alpha})}\left(\varkappa_{1},\left(\frac{\varkappa_{1}^{p}+\varkappa_{2}^{p}}{2}\right)^{1 / p} ; s, p\right)\right)^{\frac{1}{s}}\right. \\
& \quad \times\left[\frac{2^{\dot{\alpha}} \Gamma(1+\dot{\alpha})}{\Gamma(1+2 \dot{\alpha})} \mathbb{A}^{(\dot{\alpha})}\left(\left|\mathfrak{p}\left(\varkappa_{1}\right)\right|^{q},\left|\mathfrak{p}\left(\frac{\varkappa_{1}^{p}+\varkappa_{2}^{p}}{2}\right)^{1 / p}\right|^{q}\right)\right]^{\frac{1}{q}}
\end{aligned}
$$




$$
\begin{aligned}
& +\left(\Lambda_{8}^{(\dot{\alpha})}\left(\left(\frac{\varkappa_{1}^{p}+\varkappa_{2}^{p}}{2}\right)^{1 / p}, \varkappa_{2} ; s, p\right)\right)^{\frac{1}{s}} \\
& \left.\times\left[\frac{2^{\dot{\alpha}} \Gamma(1+\dot{\alpha})}{\Gamma(1+2 \dot{\alpha})} \mathbb{A}^{(\dot{\alpha})}\left(\left|\mathfrak{p}\left(\frac{\varkappa_{1}^{p}+\varkappa_{2}^{p}}{2}\right)^{1 / p}\right|^{q},\left|\mathfrak{p}\left(\varkappa_{2}\right)\right|^{q}\right)\right]^{\frac{1}{q}}\right]
\end{aligned}
$$

where $\Lambda_{7}^{(\dot{\alpha})}\left(\varkappa_{1},\left(\frac{\varkappa_{1}^{p}+\varkappa_{2}^{p}}{2}\right)^{1 / p} ; s, p\right)$ and $\Lambda_{8}^{(\dot{\alpha})}\left(\left(\frac{\varkappa_{1}^{p}+\varkappa_{2}^{p}}{2}\right)^{1 / p}, \varkappa_{2} ; s, p\right)$ are given in (3.18) and (3.19), respectively.

\subsection{Generalized special means}

For $\varkappa_{1}<\varkappa_{2}$ and $\varkappa_{1}, \varkappa_{2} \in \mathbb{R}^{\dot{\alpha}}$, considering the following $\dot{\alpha}$-type special means [72-80].

I. The generalized arithmetic mean:

$$
\mathcal{A}_{\dot{\alpha}}\left(\varkappa_{1}, \varkappa_{2}\right):=\left(\frac{\varkappa_{1}+\varkappa_{2}}{2}\right)^{\dot{\alpha}}=\frac{\varkappa_{1}^{\dot{\alpha}}+\varkappa_{2}^{\dot{\alpha}}}{2^{\dot{\alpha}}} .
$$

II. The generalized weighted arithmetic mean:

$$
\mathcal{A}_{\dot{\alpha}}\left(\varkappa_{1}, \varkappa_{2} ; w_{1}, w_{2}\right):=\frac{w_{1}^{\dot{\alpha}} \varkappa_{1}^{\dot{\alpha}}+w_{2}^{\dot{\alpha}} \varkappa_{2}^{\dot{\alpha}}}{w_{1}^{\dot{\alpha}}+w_{2}^{\dot{\alpha}}} \quad\left(w_{1}^{\dot{\alpha}}, w_{2}^{\dot{\alpha}} \in \mathbb{R}^{\dot{\alpha}}\right) .
$$

III. The generalized logarithmic mean:

$$
\mathcal{L}_{\dot{\alpha}}\left(\varkappa_{1}, \varkappa_{2}\right):=\left[\frac{\Gamma(1+n \dot{\alpha})}{\Gamma(1+(n+1) \dot{\alpha})} \frac{\varkappa_{2}^{\dot{\alpha}+1}-\varkappa_{1}^{\dot{\alpha}+1}}{\left(\varkappa_{2}-\varkappa_{1}\right)^{\dot{\alpha}}}\right] \quad\left(n \in \mathbb{Z} \backslash\{-1,0\} ; \varkappa_{1}, \varkappa_{2} \in \mathbb{R}, \varkappa_{1} \neq \varkappa_{2}\right) .
$$

Let $\chi(u)=u^{n \dot{\alpha}}(u \in \mathbb{R}: n \in \mathbb{Z},|n| \geq 2)$. Then Theorems 3.7 and 3.9 lead to Propositions 4.3 and 4.4 immediately.

Proposition 4.3 Let $\varkappa_{1}, \varkappa_{2} \in \mathbb{R}$ with $\varkappa_{1}<\varkappa_{2}, 0 \notin\left[\varkappa_{1}, \varkappa_{2}\right], p \in \mathbb{R} \backslash\{0\}$, and $n \in \mathbb{N} \backslash\{1\}$.

Then

$$
\begin{aligned}
& \left|\left[\mathcal{A}_{\dot{\alpha}}^{n}\left(\varkappa_{1},\left(\frac{\varkappa_{1}^{p}+\varkappa_{2}^{p}}{2}\right)^{\frac{1}{p}}, \varkappa_{2} ; 1^{\dot{\alpha}}, 4^{\dot{\alpha}}, 1^{\dot{\alpha}}\right)\right]-(2 p)^{\dot{\alpha}} \Gamma(1+\dot{\alpha}) \mathcal{L}_{\dot{\alpha}(p+(n-1))}\right| \\
& \leq\left(\frac{\varkappa_{2}^{p}-\varkappa_{1}^{p}}{4 p}\right)^{\dot{\alpha}}\left[( \Lambda _ { 7 } ^ { ( \dot { \alpha } ) } ( \varkappa _ { 1 } , ( \frac { \varkappa _ { 1 } ^ { p } + \varkappa _ { 2 } ^ { p } } { 2 } ) ^ { 1 / p } ; s , p ) ) ^ { \frac { 1 } { s } } \left[\frac{2^{\dot{\alpha}} \Gamma(1+\dot{\alpha})}{\Gamma(1+2 \dot{\alpha})}\right.\right. \\
& \left.\quad \times \mathbb{A}^{(\dot{\alpha})}\left(\frac{\Gamma(1+n \dot{\alpha})}{\Gamma(1+(n-1) \dot{\alpha})}\left|\varkappa_{1}^{q \dot{\alpha}(n-1)}\right|, \frac{\Gamma(1+n \dot{\alpha})}{\Gamma(1+(n-1) \dot{\alpha})}\left|\left(\frac{\varkappa_{1}^{p}+\varkappa_{2}^{p}}{2}\right)^{\dot{\alpha} q(n-1) / p}\right|\right)\right]^{\frac{1}{q}} \\
& \quad+\left(\Lambda_{8}^{(\dot{\alpha})}\left(\left(\frac{\varkappa_{1}^{p}+\varkappa_{2}^{p}}{2}\right)^{1 / p}, \varkappa_{2} ; s, p\right)\right)^{\frac{1}{s}}\left[\frac{2^{\dot{\alpha}} \Gamma(1+\dot{\alpha})}{\Gamma(1+2 \dot{\alpha})}\right. \\
& \left.\left.\quad \times \mathbb{A}^{(\dot{\alpha})}\left(\frac{\Gamma(1+n \dot{\alpha})}{\Gamma(1+(n-1) \dot{\alpha})}\left|\left(\frac{\varkappa_{1}^{p}+\varkappa_{2}^{p}}{2}\right)^{q \dot{\alpha}(n-1) / p}\right|, \frac{\Gamma(1+n \dot{\alpha})}{\Gamma(1+(n-1) \dot{\alpha})}\left|\varkappa_{2}^{q \dot{\alpha}(n-1)}\right|\right)\right]^{\frac{1}{q}}\right],
\end{aligned}
$$

where $\Lambda_{7}^{(\dot{\alpha})}\left(\varkappa_{1},\left(\frac{\varkappa_{1}^{p}+\varkappa_{2}^{p}}{2}\right)^{1 / p} ; s, p\right)$ and $\Lambda_{8}^{(\dot{\alpha})}\left(\left(\frac{\varkappa_{1}^{p}+\varkappa_{2}^{p}}{2}\right)^{1 / p}, \varkappa_{2} ; s, p\right)$ are given in (3.18) and (3.19), respectively. 
Proposition 4.4 Let $\varkappa_{1}, \varkappa_{2} \in \mathbb{R}$ with $\varkappa_{1}<\varkappa_{2}, 0 \notin\left[\varkappa_{1}, \varkappa_{2}\right], p \in \mathbb{R} \backslash\{0\}$ and $n \in \mathbb{N} \backslash\{1\}$. Then

$$
\begin{aligned}
& \left|\left[\mathcal{A}_{\dot{\alpha}}^{n}\left(\varkappa_{1},\left(\frac{\varkappa_{1}^{p}+\varkappa_{2}^{p}}{2}\right)^{\frac{1}{p}}, \varkappa_{2} ; 1^{\dot{\alpha}}, 4^{\dot{\alpha}}, 1^{\dot{\alpha}}\right)\right]-(2 p)^{\dot{\alpha}} \Gamma(1+\dot{\alpha}) \mathcal{L}_{\dot{\alpha}(p+(n-1)) \mid}\right| \\
& \leq\left(\frac{\varkappa_{2}^{p}-\varkappa_{1}^{p}}{4 p}\right)^{\dot{\alpha}}\left[\left[\frac{\Gamma(1+\dot{\alpha} s)}{\Gamma(1+(s+1) \dot{\alpha})}\right]^{\frac{1}{s}}\right. \\
& \quad \times\left[\Theta_{1}^{(\dot{\alpha})}\left(\varkappa_{1},\left(\frac{\varkappa_{1}^{p}+\varkappa_{2}^{p}}{2}\right)^{1 / p} ; q, p\right) \frac{\Gamma(1+n \dot{\alpha})}{\Gamma(1+(n-1) \dot{\alpha})}\left|\varkappa_{1}^{q \dot{\alpha}(n-1)}\right|\right. \\
& \left.\quad+\Theta_{2}^{(\dot{\alpha})}\left(\varkappa_{1},\left(\frac{\varkappa_{1}^{p}+\varkappa_{2}^{p}}{2}\right)^{1 / p} ; q, p\right) \frac{\Gamma(1+n \dot{\alpha})}{\Gamma(1+(n-1) \dot{\alpha})}\left|\left(\frac{\varkappa_{1}^{p}+\varkappa_{2}^{p}}{2}\right)^{q \dot{\alpha}(n-1) / p}\right|\right]^{\frac{1}{q}} \\
& \quad+\left[\frac{\Gamma(1+\dot{\alpha} s)}{\Gamma(1+(s+1) \dot{\alpha})}\right]^{\frac{1}{s}}\left[\Theta_{3}^{(\dot{\alpha})}\left(\left(\frac{\varkappa_{1}^{p}+\varkappa_{2}^{p}}{2}\right)^{1 / p}, \varkappa_{2} ; q, p\right)\right. \\
& \quad \times \frac{\Gamma(1+n \dot{\alpha})}{\Gamma(1+(n-1) \dot{\alpha})}\left|\left(\frac{\varkappa_{1}^{p}+\varkappa_{2}^{p}}{2}\right)^{q \dot{\alpha}(n-1) / p}\right| \\
& \left.\left.\quad+\Theta_{4}^{(\dot{\alpha})}\left(\left(\frac{\varkappa_{1}^{p}+\varkappa_{2}^{p}}{2}\right)^{1 / p}, \varkappa_{2} ; q, p\right)\right]^{\frac{1}{q}}\left|\varkappa_{2}^{q \dot{\alpha}(n-1)}\right|\right],
\end{aligned}
$$

where $\Theta_{1}^{(\dot{\alpha})}\left(\varkappa_{1},\left(\frac{\varkappa_{1}^{p}+\varkappa_{2}^{p}}{2}\right)^{1 / p} ; q, p\right), \quad \Theta_{2}^{(\dot{\alpha})}\left(\varkappa_{1},\left(\frac{\varkappa_{1}^{p}+\varkappa_{2}^{p}}{2}\right)^{1 / p} ; q, p\right), \Theta_{3}^{(\dot{\alpha})}\left(\left(\frac{\varkappa_{1}^{p}+\varkappa_{2}^{p}}{2}\right)^{1 / p}, \varkappa_{2} ; q, p\right)$ and $\Theta_{4}^{(\dot{\alpha})}\left(\left(\frac{\varkappa_{1}^{p}+\varkappa_{2}^{p}}{2}\right)^{1 / p}, \varkappa_{2} ; q, p\right)$ are given in (3.27)-(3.30), respectively.

\section{Conclusion}

In this article, we have obtained several Simpson-type inequalities for the generalized $p$ convex functions via the local fractional calculus, our results include a large number of particular cases of the generalized convexity of segmental type and generalized harmonically convex functions. Our ideas and techniques used to obtain the main results in this paper may generate new results within other classes of generalized convexity, which are not included in generalized $p$-convexity type. These types of results are useful in all the pure and applied domains of science and technique when approximation schemes are involved. It is worth mentioning that many significant real-life problems are generally defined in fractal spaces. Therefore, it is of interest to extend our results to the inequality theory, fuzzy sets and systems and machine learning, which is more general than the current branch of mathematics.

\section{Acknowledgements}

The authors would like to express their sincere thanks to the support of National Natural Science Foundation of China.

\section{Funding}

This work was supported by the National Natural Science Foundation of China (Grant No. 61673169).

Availability of data and materials

Not applicable.

Competing interests

The authors declare that they have no competing interests. 


\section{Author details}

'Department of Mathematics and General Sciences, Prince Sultan University, Riyadh, Saudi Arabia. ${ }^{2}$ Department of Medical Research, China Medical University, Taichung, Taiwan. ${ }^{3}$ Department of Computer Science and Information Engineering, Asia University, Taichung, Taiwan. ${ }^{4}$ Department of Mathematics, Government College University, Faisalabad, Pakistan. ${ }^{5}$ Département de Mathématiques, Faculté des Sciences et Techniques, Université Moulay Ismail, Errachidia, Morocco. ${ }^{6}$ Department of Mathematics, Faculty of Arts and Sciences, Giresun University, Giresun, Turkey. ${ }^{7}$ Department of Mathematics, Huzhou University, Huzhou, China. ${ }^{8}$ Hunan Provincial Key Laboratory of Mathematical Modeling and Analysis in Engineering, Changsha University of Science \& Technology, Changsha, China.

\section{Publisher's Note}

Springer Nature remains neutral with regard to jurisdictional claims in published maps and institutional affiliations.

Received: 13 May 2020 Accepted: 8 September 2020 Published online: 16 September 2020

\section{References}

1. Dragomir, S.S., Agarwal, R.P., Cerone, P.: On Simpson's inequality and applications. J. Inequal. Appl. 5(6), 533-579 (2000)

2. Liu, Z:: An inequality of Simpson type. Proc. R. Soc. Lond., Ser. A, Math. Phys. Eng. Sci. 461(2059), 2155-2158 (2005)

3. Park, J: Generalization of some Simpson-like type inequalities via differentiable s-convex mappings in the second sense. Int. J. Math. Math. Sci. 2011, Article ID 493531 (2011)

4. Set, E., Akdemir, A.O., Özdemir, M.E.: Simpson type integral inequalities for convex functions via Riemann-Liouville integrals. Filomat 31(14), 4415-4420 (2017)

5. Chu, Y.-M., Awan, M.U., Javad, M.Z., Khan, A.W.: Bounds for the remainder in Simpson's inequality via $n$-polynomial convex functions of higher order using Katugampola fractional integrals. J. Math. 2020, Article ID 4189036 (2020)

6. Yan, P.-Y., Li, Q., Chu, Y.-M., Mukhtar, S., Waheed, S.: On some fractional integral inequalities for generalized strongly modified $h$-convex function. AIMS Math. 5(6), 6620-6638 (2020)

7. Rashid, S., İscan, I., Baleanu, D., Chu, Y.-M.: Generation of new fractional inequalities via $n$ polynomials s-type convexity with applications. Adv. Differ. Equ. 2020, Article ID 264 (2020)

8. Adil Khan, M., Hanif, M., Khan, Z.A., Ahmad, K., Chu, Y.-M.: Association of Jensen's inequality for s-convex function with Csiszár divergence. J. Inequal. Appl. 2019, Article ID 162 (2019)

9. Rashid, S., Ashraf, R., Noor, M.A., Noor, K.I., Chu, Y.-M.: New weighted generalizations for differentiable exponentially convex mapping with application. AIMS Math. 5(4), 3525-3546 (2020)

10. Khan, S., Adil Khan, M., Chu, Y.-M.: Converses of the Jensen inequality derived from the Green functions with applications in information theory. Math. Methods Appl. Sci. 43(5), 2577-2587 (2020)

11. Awan, M.U., Akhtar, N., Iftikhar, S., Noor, M.A., Chu, Y.-M.: New Hermite-Hadamard type inequalities for n-polynomial harmonically convex functions. J. Inequal. Appl. 2020, Article ID 125 (2020)

12. Kalsoom, H., Idrees, M., Baleanu, D., Chu, Y.-M.: New estimates of $q_{1} q_{2}$-Ostrowski-type inequalities within a class of n-polynomial prevexity of function. J. Funct. Spaces 2020, Article ID 3720798 (2020)

13. Du, T.-S., Li, Y.-J., Yang, Z.-Q.: A generalization of Simpson's inequality via differentiable mapping using extended $(s, m)$-convex functions. Appl. Math. Comput. 293, 358-369 (2017)

14. Hsu, K.-C.. Hwang, S.-R., Tseng, K.-L.: Some extended Simpson-type inequalities and applications. Bull. Iranian Math. Soc. 43(2), 409-425 (2017)

15. Matłoka, M.: Weighted Simpson type inequalities for $h$-convex functions. J. Nonlinear Sci. Appl. 10(11), 5770-5780 (2017)

16. Shen, J.-M., Rashid, S., Noor, M.A., Ashraf, R., Chu, Y.-M.: Certain novel estimates within fractional calculus theory on time scales. AlMS Math. 5(6), 6073-6086 (2020)

17. Rashid, S., Jarad, F., Chu, Y.-M.: A note on reverse Minkowski inequality via generalized proportional fractional integral operator with respect to another function. Math. Probl. Eng. 2020, Article ID 7630260 (2020)

18. Rashid, S., Hammouch, Z., Jarad, F., Chu, Y.-M.: New estimates of integral inequalities via generalized proportional fractional integral operator with respect to another function. Fractals 28(8), Article ID 2040027 (2020)

19. Rashid, S., Khalid, A., Rahman, S., Nisar, K.S., Chu, Y.-M.: On new modifications governed by quantum Hahn's integral operator pertaining to fractional calculus. J. Funct. Spaces 2020, Article ID 8262860 (2020)

20. Ge-JiLe, H., Rashid, S., Noor, M.A., Suhail, A., Chu, Y.-M.: Some unified bounds for exponentially tgs-convex functions governed by conformable fractional operators. AIMS Math. 5(6), 6108-6123 (2020)

21. Awan, M.U., Akhtar, N., Kashuri, A., Noor, M.A., Chu, Y.-M.: $2 D$ approximately reciprocal $\rho$-convex functions and associated integral inequalities. AIMS Math. 5(5), 4662-4680 (2020)

22. Abbas Baloch, I., Mughal, A.A., Chu, Y.-M., Haq, A.U., De La Sen, M.: A variant of Jensen-type inequality and related results for harmonic convex functions. AlMS Math. 5(6), 6404-6418 (2020)

23. Awan, M.U., Talib, S., Noor, M.A., Chu, Y.-M., Noor, K...: Some trapezium-like inequalities involving functions having strongly $n$-polynomial preinvexity property of higher order. J. Funct. Spaces 2020, Article ID 9154139 (2020)

24. Zhou, S.-S., Rashid, S., Jarad, F., Kalsoom, H., Chu, Y.-M.: New estimates considering the generalized proportional Hadamard fractional integral operators. Adv. Differ. Equ. 2020, Article ID 275 (2020)

25. Rashid, S., Akdemir, A.O., Jarad, F., Noor, M.A., Noor, K.I.: Simpson's type integral inequalities for $\boldsymbol{\kappa}$-fractional integrals and their applications. AIMS Math. 4(4), 1087-1100 (2019)

26. Li, Y.-J., Du, T.: Some Simpson's type integral inequalities for functions whose third derivatives are $(\dot{\alpha}, m)-G A-c o n v e x$ function. J. Egypt. Math. Soc. 24, 175-180 (2016)

27. Xi, B.-Y., Qi, F.: Integral inequalities of Simpson type for logarithmically convex functions. Adv. Stud. Contemp. Math. 23(4), 559-566 (2013)

28. Sarikaya, M.Z., Set, E., Özdemir, M.: On new inequalities of Simpson's type for s-convex functions. Comput. Math. Appl. 60(8), 2191-2199 (2010) 
29. İscan, I., Konuk, N.K., Kadakal, M.: Some new Simpson type inequalities for the $p$-convex and $p$-concave functions. Commun. Fac. Sci. Univ. Ank. Sér. A1 Math. Stat. 67(2), 252-263 (2018)

30. Khurshid, Y., Adil Khan, M., Chu, Y.-M.: Conformable fractional integral inequalities for GG- and GA-convex functions. AlMS Math. 5(5), 5012-5030 (2020)

31. Xu, L., Chu, Y.-M., Rashid, S., El-Deeb, A.A., Nisar, K.S.: On new unified bounds for a family of functions with fractional q-calculus theory. J. Funct. Spaces 2020, Article ID 4984612 (2020)

32. Rashid, S., Hammouch, Z., Baleanu, D., Chu, Y.-M.: New generalizations in the sense of the weighted non-singular fractional integral operator. Fractals 28(7), Article ID 2040003 (2020)

33. Zhao, T.-H., He, Z.-Y., Chu, Y.-M.: On some refinements for inequalities involving zero-balanced hypergeometric function. AlMS Math. 5(6), 6479-6495 (2020)

34. Agarwal, P., Kadakal, M., İscan, I., Chu, Y.-M.: Better approaches for $n$-times differentiable convex functions. Mathematics 8, Article ID 950 (2020)

35. Wang, M.-K., Chu, H.-H., Li, Y.-M., Chu, Y.-M.: Answers to three conjectures on convexity of three functions involving complete elliptic integrals of the first kind. Appl. Anal. Discrete Math. 14(1), 255-271 (2020)

36. Shen, J.-M., Yang, Z.-H., Qian, W.-M., Zhang, W., Chu, Y.-M.: Sharp rational bounds for the gamma function. Math. Inequal. Appl. 23(3), 843-853 (2020)

37. Wang, M.-K., Chu, Y.-M., Li, Y.-M., Zhang, W.: Asymptotic expansion and bounds for complete elliptic integrals. Math Inequal. Appl. 23(3), 821-841 (2020)

38. Hai, G.-J., Zhao, T.-H.: Monotonicity properties and bounds involving the two-parameter generalized Grötzsch ring function. J. Inequal. Appl. 2020, Article ID 66 (2020)

39. Sun, M.-B., Chu, Y.-M.: Inequalities for the generalized weighted mean values of $g$-convex functions with applications. Rev. R. Acad. Cienc. Exactas Fís. Nat., Ser. A Mat. 114(4), Article ID 172 (2020)

40. Adil Khan, M., Pečarić, J., Chu, Y.-M.: Refinements of Jensen's and McShane's inequalities with applications. AIMS Math. 5(5), 4931-4945 (2020)

41. Zhao, T.-H., Wang, M.-K., Chu, Y.-M.: A sharp double inequality involving generalized complete elliptic integral of the first kind. AIMS Math. 5(5), 4512-4528 (2020)

42. Zhao, T.-H., He, Z.-Y., Chu, Y.-M.: On some refinements for inequalities involving zero-balanced hypergeometric function. AlMS Math. 5(6), 6479-6495 (2020)

43. Awan, M.U., Talib, S., Chu, Y.-M., Noor, M.A., Noor, K.I.: Some new refinements of Hermite-Hadamard-type inequalities involving $\Psi_{k}$-Riemann-Liouville fractional integrals and applications. Math. Probl. Eng. 2020, Article ID 3051920 (2020)

44. Yang, X.-Z., Farid, G., Nazeer, W., Chu, Y.-M., Dong, C.-F.: Fractional generalized Hadamard and Fejér-Hadamard inequalities for $m$-convex function. AIMS Math. 5(6), 6325-6340 (2020)

45. Guo, S.-Y., Chu, Y.-M., Farid, G., Mehmood, S., Nazeer, W.: Fractional Hadamard and Fejér-Hadamard inequaities associated with exponentially (s, m)-convex functions. J. Funct. Spaces 2020, Article ID 2410385 (2020)

46. Iqbal, A., Adil Khan, M., Ullah, S., Chu, Y.-M.: Some new Hermite-Hadamard-type inequalities associated with conformable fractional integrals and their applications. J. Funct. Spaces 2020, Article ID 9845407 (2020)

47. Khurshid, Y., Adil Khan, M., Chu, Y.-M.: Conformable integral version of Hermite-Hadamard-Fejér inequalities via $\eta$-convex functions. AIMS Math. 5(5), 5106-5120 (2020)

48. Qi, H.-X., Yussouf, M., Mehmood, S., Chu, Y.-M., Farid, G.: Fractional integral versions of Hermite-Hadamard type inequality for generalized exponentially convexity. AIMS Math. 5(6), 6030-6042 (2020)

49. Iqbal, A., Adil Khan, M., Mohammad, N., Nwaeze, E.R., Chu, Y.-M.: Revisiting the Hermite-Hadamard integral inequality via a Green function. AlMS Math. 5(6), 6087-6107 (2020)

50. Rashid, S., Safdar, F., Akdemir, A.O., Noor, M.A., Noor, K...: Some new fractional integral inequalities for exponentially m-convex functions via extended generalized Mittag-Leffler function. J. Inequal. Appl. 2019, Article ID 299 (2019)

51. Rashid, S., Jarad, F., Noor, M.A., Kalsoom, H., Chu, Y.-M.: Inequalities by means of generalized proportional fractional integral operators with respect to another function. Mathematics 7(12), Article ID 1225 (2019)

52. Yang, Y.-J., Baleanu, D., Yang, X.-J: Analysis of fractal wave equations by local fractional Fourier series method. Adv. Math. Phys. 2013, Article ID 632309 (2013)

53. Yang, X.-J., Baleanu, D., Srivastava, H.M.: Local Fractional Integral Transforms and Their Applications. Elsevier/Academic Press, Amsterdam (2016)

54. Julia, G.: Mémoire sur l'itération des fonctions rationnelles. J. Math. Pures Appl. 4, 47-245 (1918)

55. Kwun, Y.C., Shahid, A.A., Nazeer, W., Abbas, M., Kang, S.M.: Fractel generation via CR iteration scheme with s-convexity. IEEE Access 7, 69986-69997 (2019)

56. Kumari, S., Kumari, M., Chugh, R.: Generation of new fractals via SP orbit with s-convexity. Int. J. Eng. Technol. 9(3), 2491-2504 (2017)

57. Mo, H.-X., Sui, X.: Hermite-Hadamard-type inequalities for generalized s-convex functions on real linear fractal set $\mathbb{R}^{\alpha}$ $(0<\alpha<1)$. Math. Sci. 11(3), 241-246 (2017)

58. Mo, H.-X., Sui, X., Yu, D.-Y.: Generalized convex functions on fractal sets and two related inequalities. Abstr. Appl. Anal. 2014, Article ID 636751 (2014)

59. Mo, H.-X.: Generalized Hermite-Hadamard type inequalities involving local fractional integrals. https://arxiv.org/ftp/arxiv/papers/1410/1410.1062.pdf. arXiv:1410.1062 [math.CA]

60. Chen, G.-S.: Generalizations of Hölder's and some related integral inequalities on fractal space. J. Funct. Spaces Appl. 2013, Article ID 198405 (2013)

61. Du, T.-S., Wang, H., Adil Khan, M., Zhang, Y.: Certain integral inequalities considering generalized $m$-convexity on fractal sets and their applications. Fractals 27(7), Article ID 1950117 (2019)

62. Luo, C.-Y., Wang, H., Du, T.-S.: Fejér-Hermite-Hadamard type inequalities involving generalized h-convexity on fractal sets and their applications. Chaos Solitons Fractals 131, Article ID 109547 (2020)

63. Sarikaya, M.Z., Budak, H., Erden, S.: On new inequalities of Simpson's type for generalized convex functions. Korean J. Math. 27(2), 279-295 (2019)

64. Vivas, M., Hernández, J., Merentes, N.: New Hermite-Hadamard and Jensen type inequalities for h-convex functions on fractal sets. Rev. Colomb. Mat. 50(2), 143-162 (2016) 
65. Sarikaya, M.Z., Budak, H.: Generalized Ostrowski type inequalities for local fractional integrals. Proc. Am. Math. Soc. 145(4), 1527-1538 (2017)

66. Sun, W.-B.: On generalization of some inequalities for generalized harmonically convex functions via local fractional integrals. Quaest. Math. 42(9), 1159-1183 (2019)

67. Budak, H., Sarikaya, M.Z., Yildirim, H.: New inequalities for local fractional integrals. Iran. J. Sci. Technol. Trans. A, Sci. 41(4), 1039-1046 (2017)

68. Erden, S., Sarikaya, M.Z., Çelik, N.: Some generalized inequalities involving local fractional integrals and their applications for random variables and numerical integration. J. Appl. Math. Stat. Inform. 12(2), 49-65 (2016)

69. Zhang, K.-S., Wan, J.-P.: p-Convex functions and their properties. Pure Appl. Math. 23(1), 130-133 (2007)

70. İscan, i.: Hermite-Hadamard type inequalities for harmonically convex functions. Hacet. J. Math. Stat. 43(6), 935-942 (2014)

71. Işcan, i.: New estimates on generalization of some integral inequalities for $s$-convex functions and their applications. https://arxiv.org/pdf/1207.7114.pdf. arXiv:1207.7114 [math.CA]

72. Pearce, C.E.M., Pečarić, J.: Inequalities for differentiable mappings with application to special means and quadrature formulae. Appl. Math. Lett. 13(2), 51-55 (2000)

73. Chu, Y.-M., Long, B.-Y.: Best possible inequalities between generalized logarithmic mean and classical means. Abstr. Appl. Anal. 2010, Article ID 303286 (2020)

74. Chu, Y.-M., Xia, W.-F.: Two optimal double inequalities between power mean and logarithmic mean. Comput. Math. Appl. 60(1), 83-89 (2010)

75. Zhang, X.-M., Chu, Y.-M.: Convexity of the integral arithmetic mean of a convex function. Rocky Mt. J. Math. 40(3), 1061-1068 (2010)

76. Wang, M.-K., Chu, Y.-M., Qiu, Y.-F., Qiu, S.-L.: An optimal power mean inequality for the complete elliptic integrals. Appl. Math. Lett. 24(6), 887-890 (2011)

77. Chu, Y.-M., Wang, M.-K., Qiu, S.-L.: Optimal combinations bounds of root-square and arithmetic means for Toader mean. Proc. Indian Acad. Sci. Math. Sci. 122(1), 41-51 (2012)

78. Chu, Y.-M., Wang, M.-K.: Optimal Lehmer mean bounds for the Toader mean. Results Math. 61(3-4), 223-229 (2012)

79. Qian, W.-M., Zhang, X.-H., Chu, Y.-M.: Sharp bounds for the Toader-Qi mean in terms of harmonic and geometric means. J. Math. Inequal. 11(1), 121-127 (2017)

80. Qian, W.-M., Chu, Y.-M.: Sharp bounds for a special quasi-arithmetic mean in terms of arithmetic and geometric means with two parameters. J. Inequal. Appl. 2017, Article ID 274 (2017)

\section{Submit your manuscript to a SpringerOpen ${ }^{\circ}$ journal and benefit from:}

- Convenient online submission

- Rigorous peer review

- Open access: articles freely available online

- High visibility within the field

- Retaining the copyright to your article

Submit your next manuscript at $\gg$ springeropen.com 